\title{
A Comparison of Global Magnetic Field Skeletons and Active-Region Upflows
}

\author{
S. J. Edwards ${ }^{1,2}$. C. E. Parnell ${ }^{2}$. \\ L. K. Harra ${ }^{3}$. J. L. Culhane ${ }^{3}$. \\ D. H. Brooks ${ }^{4,5}$
}

(C) Springer $\bullet \bullet \bullet \bullet$

\begin{abstract}
Plasma upflows have been detected in active regions using Doppler velocity maps. The origin and nature of these upflows is not well known with many of their characteristics determined from the examination of single events. In particular, some studies suggest these upflows occur along open-field lines and, hence, are linked to sources of the solar wind. To investigate the relationship these upflows may have with the solar wind, and to probe what may be driving them, this paper considers seven active regions observed on the solar disc using the Extreme ultraviolet Imaging Spectrometer aboard Hinode between August 2011 and September 2012. Plasma upflows are observed in all these active regions. The locations of these upflows are compared to the global potential magnetic field extrapolated from the Solar Dynamics Observatory, Helioseismic and Magnetic Imager daily synoptic magnetogram taken on the day the upflows were observed. The structure of the magnetic field is determined by constructing its magnetic skeleton in order to help identify open-field regions and also sites where magnetic reconnection at global features is likely to occur. As a further comparison, measurements of the temperature, density and composition of the plasma are taken from regions with active-region upflows. In most cases the locations of the upflows in the active regions do not correspond to areas of open field, as predicted by a global coronal potential field model, and therefore these upflows are not always sources of the slow solar wind. The locations of the upflows are, in general, intersected by separatrix surfaces associated with null points located high in the corona; these could be important sites of reconnection with global consequences.
\end{abstract}

\footnotetext{
${ }^{1}$ Department of Mathematical Sciences, University of Durham, Durham, UK

${ }^{2}$ University of St Andrews, School of Mathematics \& Statistics, North Haugh, St Andrews, Fife, Scotland, UK, KY16 9SS email:cep@st-andrews.ac.uk

${ }^{3}$ UCL-Mullard Space Science Laboratory, Holmbury St Mary, Dorking, Surrey, UK, RH5 6NT

${ }^{4}$ College of Science, George Mason University, 4400

University Drive, Fairfax, VA 22030

${ }^{5}$ Current address: Hinode Team, ISAS/JAXA, 3-1-1

Yoshinodai, Chuo-ku, Sagamihara, Kanagawa 252-5210,

Japan
} 
Keywords: Solar wind; Magnetic fields, Corona

\section{Introduction}

One of the significant discoveries from the Hinode EUV Imaging Spectrometer (EIS; Culhane et al., 2007) instrument is the observation of persistent upflows at edges of active regions (ARs). These had been observed in the preHinode era with Transition Region and Coronal Explorer (TRACE; Handy et al., 1999) images, and with it Solar Ultraviolet Measurements of Emmited Radiation (SUMER; Wilhelm et al., 1995) onboard Solar and Heliospheric Observatory (SOHO). However, with Hinode these upflows are now observed regularly spectroscopically, and are seen in some form in every AR observed. The upflows tend to occur in regions of low intensity. The first Hinode example of these flows used a combination of imaging data, from the X-ray telescope, and spectroscopic data, from EIS (Sakao et al., 2007). The AR observed was located close to a coronal hole $(\mathrm{CH})$ and showed persistent flowing plasma in the X-ray images, around fan-like magnetic field lines with speeds reaching over $100 \mathrm{~km} \mathrm{~s}^{-1}$. When observed using imaging data there can be some dispute as to whether these are true upflows, but this was confirmed with EIS spectroscopic data. The plasma was significantly blue-shifted in the fan-like structures. It was estimated that outflowing plasma from such locations could supply around $25 \%$ of the mass of the slow solar wind, reinforcing the importance of understanding the formation of this outflow.

Doschek et al. (2008) analysed two ARs to determine various physical parameters such as temperature, density, non-thermal velocity. They found that the upflow regions have temperatures of around $1.3 \mathrm{MK}$, with a low density of $7 \times 10^{8}$ $\mathrm{cm}^{-3}$. There is a positive correlation between the Doppler velocity and the nonthermal velocity indicating that a range of velocities exists in these regions. Hara et al. (2008) found deviations from a single Gaussian profile in some regions with a strong blue shift indicating that there are unresolved high-speed flows. Bryans, Young, and Doschek (2010) imposed a two-component Gaussian fit on the profiles and found speeds reaching $200 \mathrm{~km} \mathrm{~s}^{-1}$. However the main component was still showing a correlation with the non-thermal velocity indicating unresolved additional flows. Ugarte-Urra and Warren (2011) studied the temporal evolution of the flows and found that they show blue-wing enhancements on timescales as short as the $5 \mathrm{~min}$ cadence used, so the process creating the upflows is very dynamic. These upflowing features seem to be predominately at million-degree coronal temperatures (Warren et al., 2011) whereas the cooler transition-region emission, so often seen as fan-like structures, tends to show downflows and not upflows.

The fact that these blue shifts are seen so consistently hints strongly at a link to plasma that ends up forming part of the slow solar wind. However, verifying this link is extremely difficult. The measurements from Hinode are over a small field-of-view in the corona. The solar wind is measured at 1AU. The distances involved and linking the remote sensing data to the Advanced Composition Explorer (ACE) data to confirm this is really a source of the solar wind is 
challenging. Slemzin et al. (2013) aimed to link the smaller field-of-view features seen with EIS to the large-scale structures seen as coronal rays at the limb out to more than a solar radius. For the two ARs observed, the locations of the upflows with EIS were consistent with the open magnetic field lines determined by a potential-field source-surface model, and aligned with the limb coronal rays. The in-situ data at ACE were also consistent with the AR as a source of the solar wind. Brooks and Warren (2011) measured the chemical composition of the upflowing regions in EIS and compared these to the composition data from ACE and these were found to match. In follow-up work (Brooks and Warren, 2012) they found that the blue-wing asymmetries also have a composition consistent with the slow solar-wind values.

The source of the upflows is still up for debate. Evidence has been found of a correlation between asymmetries in the coronal line profiles and the behaviour and speeds seen in chromospheric type II spicules by De Pontieu et al. (2009). This suggests that the bulk of the energy that heats the corona or forms the solar wind may come from the lower atmosphere. A study by He et al. (2010) also considered the relationship of coronal upflows with chromospheric jets and found a strong correlation. These results seem inconsistent with other work that shows that the source may more likely be in the corona. Harra et al. (2008) modelled the magnetic-field structure of an AR with strong upflows and found that the strong blue-shifted regions are caused by the expansion of loops that lie over ARs and which can reconnect with neighbouring large-scale loops or are open to interplanetary space. A comparison of simulations and observations found that in the case of emerging flux into an existing AR, the strong upflows are formed by a combination of compression and reconnection, depending on the orientation of the magnetic field (Harra et al., 2012). Upflow regions have also been found to be consistent with the locations where the magnetic field displays strong gradients of magnetic connectivity (namely quasi-separatrix layers; Baker et al., 2009). They suggest that magnetic reconnection in these regions between closed field in the AR and large-scale 'open' field outside the AR could drive the upflows. Additional support for reconnection occurring high in the corona was found by Del Zanna et al. (2011) who compared radio noise storms and coronal outflows with magnetic-field extrapolations. They suggest that the continuous growth of ARs and the close-by 'open fields' maintains a steady process of interchange reconnection occurring high in the corona, which drives the upflowing plasma. Furthermore, coronal abundance values were found by Brooks and Warren (2012) for the plasma producing the line-profile asymmetries, and this suggests that the material is trapped in closed loops before escaping, also indicating a coronal origin, which could be caused by interchange reconnection.

Of course, these upflows in ARs are always observed - but it is not clear what percentage of the upflows are still within closed loops and never make it into the slow solar wind and how much is truly open to the heliosphere. A detailed study of a time period was carried out by van Driel-Gesztelyi et al. (2012), which had two ARs and two CHs. Modelling was preformed on both global and local scales to try and understand the magnetic field set-up. Through these methods they could determine which upflows actually formed part of the slow solar wind - in the example they looked at it was the upflows of an AR that bordered a $\mathrm{CH}$. 
More recently, Culhane et al. (2014) and Mandrini et al. (2014) have reported the results of a similarly detailed study for a single AR with two upflow regions that had no directly associated CHs. While potential-field source-surface (PFSS) modelling showed no open field associated with the upflow regions, a combination of local and global magnetic field modelling suggested that a twostage reconnection process allowed upflow material to be transported to an area of open field north-west of the AR. Following a detailed back-mapping process, plasma with AR and slow solar wind characteristics originating from the west of the AR was identified by the ACE in-situ instruments several days later.

In this paper, we study a number of ARs in different time periods, with different characteristics - some are in different hemispheres, some are located next to CHs, some are not. All of them show upflowing plasma. For each AR, we first identify its main physical characteristics. Then, we carry out modelling to determine where the actual open magnetic field is, and correlate this with the observations. We are seeking to distinguish between open and closed field. If this can be done in a consistent and fast way, it would prove to be extremely valuable for understanding the source of the slow solar wind. Indeed in terms of a practical use, it could be employed as a tool for choosing regions to observe for the future Solar Orbiter mission - the purpose of which is to understand the source of the solar wind from its creation on the Sun to its propagation into the heliosphere. We also aim to shed light on whether these upflows are associated with global coronal reconnection, thus addressing the question of whether they are coronal in origin.

\section{Data Analysis: Observations and Model}

\subsection{Observational Data}

We have analysed data from EIS onboard the Hinode spacecraft (Kosugi et al., 2007). The standard calibration was used through the solarsoftware routine eis_prep. Additionally, the slit tilt and the orbital variation of line position were corrected. For each pixel we fitted the line using a single Gaussian profile, to produce an overall intensity 'image' for each raster scan. These images were then coaligned taking account of their spatial displacements as a function of wavelength. In order to determine the Doppler velocity a rest wavelength is required. We choose a region of quiet Sun or diffuse corona close to each AR raster to determine this, and used this value to produce a Doppler velocity map that shows relative velocities.

To assess differences between the characteristics of each of the ARs, we also measured the electron density $(N)$, temperature $(T)$, emission measure distribution $(E M)$, and plasma composition from the EIS data. The method we used to determine these quantities is that of Brooks and Warren (2011). Briefly, we used the Fe XIII 203.826/202.044 $\AA$ diagnostic to determine the electron density, and this measurement was then used to compute the EM distribution. For this purpose, we selected strong Fe lines covering the temperature range of formation of Fe vIII to Fe XVII (0.4-4.0 MK). To model the contribution functions 
needed for the EM determination we used the CHIANTI database (Dere et al., 1997; Landi et al., 2012) and adopted the photospheric abundances of Grevesse, Asplund, and Sauval (2007). The EM calculation itself was performed with the MCMC (Monte Carlo Markov Chain) procedure in the PINTofALE software package (Kashyap and Drake, 1998, 2000).

Once the EM distribution is known from the Fe lines, we can calculate a measure of the plasma composition by modeling the intensities of the Si X 258.375 $\AA$ and S x $264.223 \AA$ lines. The composition of the corona is enhanced compared to that of the photosphere, and one way to detect this difference is to look at the EM derived from lines from elements with low $(<10 \mathrm{eV})$ and high $(>10$ eV) first ionization potential (FIP). Since Si and Fe are low FIP elements, the EM calculated from the Fe lines should also reproduce the observed intensity of Si X $258.375 \AA$ when convolved with its contribution function. Conversely, since S is a high FIP element the predicted intensity for S X $264.223 \AA$ will be too large if the plasma composition is enhanced. Here, we use the ratio of the predicted to observed intensities for the S X $264.223 \AA$ line as our measure of the compositional enhancement in the corona (FIP bias). In cases where the $\mathrm{Si}$ X $258.375 \AA$ line was not observed, we have substituted it for the Si X 261.050 $\AA$ line. For more discussion of the FIP effect see the recent review by Laming (2014 (submitted)).

In addition, we analysed data from the Atmospheric Imaging Assembly (AIA; Lemen et al., 2012) onboard the Solar Dynamics Observatory (SDO). In order to aid the alignment between AIA and EIS, we used the $195 \AA$ emission line in both instruments and manually aligned the two data sets by means of feature recognition. We also used the SDO Helioseismic and Magnetic Imager (HMI; Scherrer et al., 2012) line-of-sight magnetograms and daily synoptic maps in order to carry out the coronal field modelling.

\subsection{Potential-Field Source-Surface Model}

A PFSS model (van Ballegooijen, Cartledge, and Priest, 1998; Mackay and Yeates, 2012) is used to determine the magnetic topology of the global coronal magnetic field at the seven different times when upflows are observed from ARs.

The PFSS model has two boundary conditions. First, the radial component of the magnetic field at the lower boundary is specified by a synoptic magnetogram. Secondly, the magnetic field at the upper boundary is fixed to be completely radial; this upper boundary is known as the source-surface.

The lower boundary for the model used here is taken from the HMI dailyupdate synoptic maps. These maps are produced from the full disc radial magnetograms (which are calculated using the 720s line-of-sight full-disc magnetograms). The $60^{\circ}$ either side of central meridian are taken from a 4 -h average of the full-disc magnetograms from that day and the remaining map is taken from the standard Carrington rotation synoptic magnetogram. Owing to the fact that a large proportion of the data from these maps is collected on a single day, the $B$-angle tilt can lead to missing data at the poles. These data gaps are filled in by using data from the Carrington rotation maps or, if that is also missing, then averaging over adjacent pixels. 
The synoptic maps have a resolution of 3600 gridpoints equally spaced in longitude and 1440 gridpoints equally spaced in sine latitude. This resolution is too high for us to sensibly apply our PFSS model and so the resolution is reduced by a factor of a half, to 1800 by 720 , in order to extrapolate the magnetic field. Since, here, we are interested in the global magnetic topology such a reduction in resolution has little effect. Specifically, it does not substantially change the locations of the open-field regions (OFRs).

The PFSS code solves Laplace's equation in terms of spherical harmonics $B_{l m}$ (van Ballegooijen, Cartledge, and Priest, 1998; Mackay and Yeates, 2012). For a perfect solution, an infinite number of harmonics would be needed, but numerical error in the small high-order harmonics leads to "spherical ringing". We use a harmonic number $l=351$ such that the resolution of the potential-field extrapolation is maximised without the introduction of spherical ringing. This produces a resolution of 1409 gridpoints equally spaced in longitude and 709 gridpoints equally spaced in latitude in the model.

In our model, the source surface is set at a radial distance of $2.0 R_{\odot}$. A total of 157 exponentially spaced gridpoints cover the distance between the solar and source surfaces. A source surface at $2.0 R_{\odot}$ is chosen instead of the usual height of $2.5 R_{\odot}$ to allow more field regions to become open, as would be expected at solar maximum when the field is further from potential.

\subsubsection{Determining the Magnetic Topology}

The magnetic topology of the global coronal field found using the PFSS model is determined by calculating, and illustrated by plotting, its magnetic skeleton. The skeleton of a magnetic field consists of the boundaries between topologically distinct flux domains within which field lines can (although do not always) evolve via smooth ideal motions. However, the only way flux can transfer from one domain to the next is via reconnection, hence, the elements of the magnetic skeleton are all possible sites for reconnection.

The standard elements of any magnetic skeleton are null points, their associated separatrix surfaces, spines and separators: the latter are special field lines linking pairs of null points. The null points are found using the trilinear null finding method (Haynes and Parnell, 2007). The spines of the nulls can be easily found by identifying the direction of the eigenvector associated with the largest eigenvalue of the Jacobi matrix of the magnetic field determined at the null point. This approach works for our magnetic field because it is potential, but will not always work for non-potential nulls.

The separatrix surfaces associated with the null points cannot be calculated so simply as the field lines within them rapidly converge towards the path of the field line associated with the major fan eigenvalue (Parnell et al., 1996), and so a line, as opposed to a surface, is typically found. To determine the full separatrix surface, the method by Haynes and Parnell (2010) is used. This method is also used to find the generic separators in the model, i.e., those separators that link opposite-polarity nulls and lie at the intersection of the separatrix surfaces from these nulls.

Finally, for global PFSS fields there are additional elements of the magnetic skeleton: the heliospheric current sheet (HCS) null line and associated HCS 
curtains. The HCS null line is located on the source surface and lies at the change between fields that are directed radially outwards and radially inwards. It lies at the base of the HCS, which resides outside the source surface at the interface between the oppositely directed radial fields. From the HCS null line two separatrix surfaces extend down, called HCS curtains, which indicate the boundary between open and closed magnetic fields within the model. Fields that reside between the two curtains are closed, whilst the magnetic field outside the HCS curtains will be open unless it is trapped within a separatrix dome (closedseparatrix structure) formed from the separatrix surfaces of one or more coronal nulls (Platten et al., 2014).

During solar maximum, which is the time period during which the ARs studied here occurred, the HCS null line may form more than one closed curve on the source surface (Wang, Young, and Muglach, 2014). This occurs because, at solar maximum, the polar fields are very weak and so they are completely dominated by active-region fields, which at this time form the Sun's open field. The HCS null lines may be located and extend anywhere, from the poles to the equator and back. For example, from the seven global fields we consider, Figure 10 shows that 29 August 2012 has three separate HCS null lines on the source surface, whilst there are two separate HCS null lines on 21 August 2011 (Figure 2), 2 March 2012 (Figure 6), 15 September 2012 (Figure 12) and 23 September 2012 (Figure 14). Note that, although highly warped and fragmented HCS null lines indicate that the solar wind can originate from anywhere on the solar surface, they do not indicate the number of distinct regions of open flux. To determine these the open separatrix curtains must also be found (e.g., for more details see Platten et al., 2014).

\section{Active Region Analysis}

We analyse seven different ARs that are located at various places on the disk, providing a variety of examples in the northern and southern hemispheres. In the following sub-sections, we describe each AR, before comparing the location(s) of the AR's blue-shifted region(s), with the global coronal potential magnetic topology, as determined by the PFSS model, in the vicinity of the observed AR.

The relative locations of ARs and CHs were established from AIA $193 \AA$ maps where AR and $\mathrm{CH}$ contours were drawn using the SPoCA-AR and SPoCA-CH algorithms (Verbeeck et al., 2014). The maps for the relevant dates and times were obtained using the Heliospheric Feature Catalogue (http://voparis-helio. obspm.fr/hfc-gui/index.php).

For each AR, we include two figures. One shows the AIA $193 \AA$, the EIS Fe XII intensity and EIS Doppler velocities and the maximum height reached by field lines from the PFSS model. The other shows the field lines and topological structures derived from the PFSS model. Also, for each AR a number of boxes are selected (highlighted in black on the doppler velocity images) in which the temperature, density and emission measure are determined. These plasma parameters are detailed in Table 1 and discussed in Section 4. 

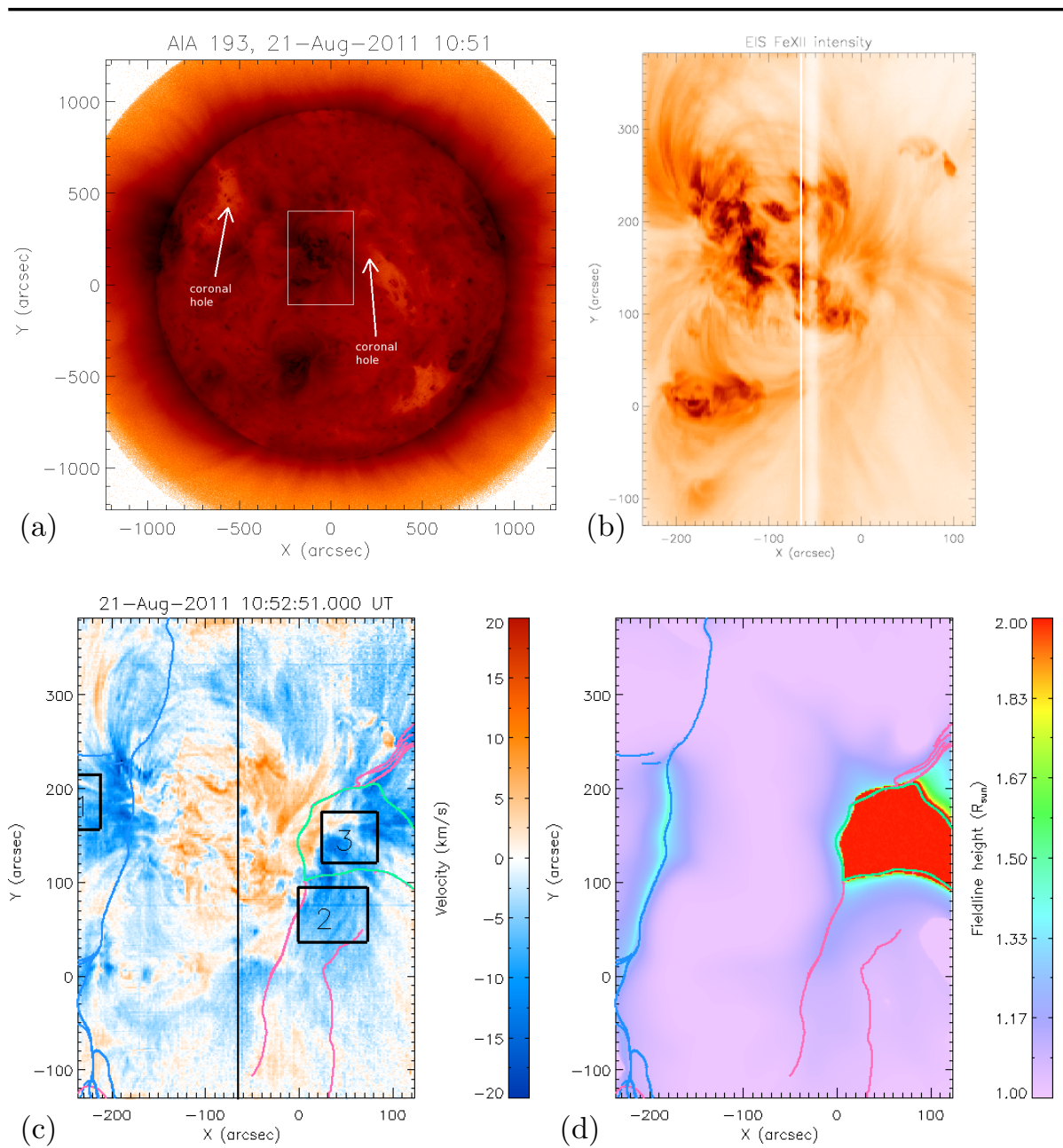

Figure 1. (a) AIA $193 \AA$ negative intensity image on 21 August for AR 11271. The white box shows the position of the EIS raster. (b) EIS negative intensity image and (c) EIS Doppler velocity map from the Fe XII emission line. Three black boxes are overlaid on the Doppler map to indicate where plasma characteristics were determined. Also overlaid on the Doppler map is the intersection with the photosphere of separatrix surfaces (pink/blue lines) from positive/negative coronal nulls and the HCS curtains (green lines) all determined from a PFSS global field model. (d) Contour map of the maximum height reached by potential field lines traced from the photosphere around AR11271.

\subsection{Active Region NOAA 11271}

Active region NOAA 11271 , located at about $17^{\circ}$ north, crossed the disc between 15 August and 28 August 2011 and was observed by both AIA (Figure 1a) and EIS (Figure 1b-1c) on 21 August with the raster starting at 10:52 UT. Its magnetic complexity increased during the disc transit, with the number of spots peaking on 23 August. During the disc passage there were 14 C-class flares and 

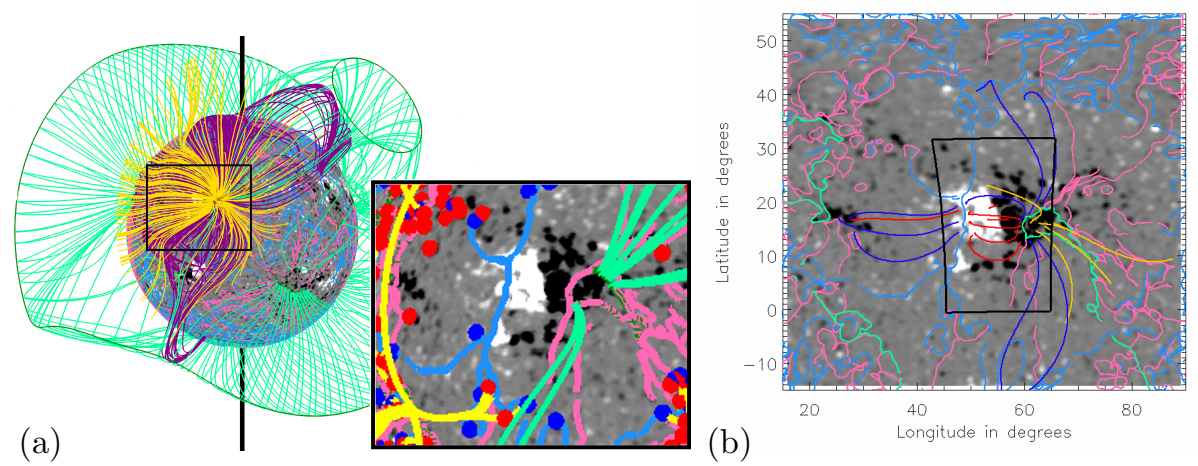

Figure 2. (a) $3 \mathrm{D}$ global potential field around AR 11271, as viewed at 10:51 on 21 August 2011. The solar surface is shaded according to the radial magnetic field component at the photosphere. The thick green lines on the source surface indicate the location of the HCS null line from which field lines lying in the HCS curtains (thin green lines) extend down. A few closed (wine) and open (yellow) field lines originating from in and around the AR are also plotted. The cut out (indicated by a black box on the global image) shows a close up of the magnetic skeleton features in the AR, including null points (positive - red, negative -blue) and separators (thick yellow lines) are identified. (b) Sample field lines (red: $<300 \mathrm{Mm}$, blue: $>300 \mathrm{Mm}$, yellow: open) in and around AR11271 overlaid on a contour plot of the photospheric magnetic field from the synoptic map for 21 August 2011. In all three graphs, light blue/pink lines show the intersection of negative/positive separatrix surfaces with the photosphere, whilst light green lines are the intersection of the HCS curtains with the photosphere. The black skewed box shows the location of the EIS field of view. Since this is on a cutout from the synoptic map the box is warped.

a larger number of B-class events. Two significant CHs were observed, east and west of the AR, but neither overlapped with the EIS field of view. The $\mathrm{CH}$ to the west closed on 22/23 August. This AR was at least 25 days old prior to the EIS observation.

From the 3D representation of the coronal field around AR11271 (Figure 2a) field lines (green lines) outlining the HCS curtains, the boundaries between open and closed field, are traced from the HCS null-line (dark green lines on source surface). Sample closed (wine) and open (yellow) field lines from the area around the AR11271 are plotted. The open field originates from a small part of the negative-polarity region slightly to the west of the AR and is probably the counterpart to the observed western $\mathrm{CH}$, although in the PFSS model it appears nearer to the AR than in the observations.

In order to compare the locations of open-field and high-reaching field lines with the upflows, we determine where the HCS curtains (which divide regions of open and closed field) and separatrix surfaces intersect with the photosphere. We also create a map of the maximum height reached by the field lines from our PFSS (Figure 1d). The intersections of the HCS curtains are overlaid on the EIS Doppler velocity maps (Figure 1c). The large OFR mentioned above is visible to the west of the AR, outlined by a green line (Figure 1c) and highlighted by the red patch in Figure 1d, lines up well with a region of upflow, suggesting that plasma from this upflowing region could contribute to the solar wind. However, 
on the east of the AR, although there is another large upflowing region, there is no corresponding OFR in the PFSS model. Instead, in Figure 1d, a cyan coloured patch of field extending up high in the atmosphere to around $1.65 R \odot$ $(440 \mathrm{Mm})$ is found. Much further to the east there is an OFR in the PFSS model that probably corresponds to the observed eastern $\mathrm{CH}$ (region on the left of Figure 2b bounded by the HCS curtains - green lines).

In Figure 2b, example field lines are plotted on a close up of the AR. The field lines are coloured according to their length: red lines are $<300 \mathrm{Mm}$, blue lines are $>300 \mathrm{Mm}$ and yellow lines are open. The locations of the longest field lines closely agree with the locations of the highest reaching field lines: these are associated with upflows. Field lines in downflow regions appear to be shorter and either connect within the AR or connect the AR to close-by weak quiet-sun field.

By comparing Figures 1c and 1d and the cut out in Figure 2a it is also possible to see whether the upflows are located at likely regions of reconnection with largescale global consequences (note, that with the resolution of our PFSS model we cannot see small-scale topological features, i.e., potential sites of reconnection low in the atmosphere). The edge of the OFR seen in the PFSS model is a potential site for interchange reconnection, furthermore, separatrix surfaces from coronal nulls (red/blue dots in the cut out), which mark the boundaries between two topologically distinct global flux regions, and separators (yellow lines in cut out) are also possible reconnection sites. Although there are no coronal nulls directly above the upflow regions, separatrix surfaces intersect these regions, suggesting that interchange reconnection between different topological regions may play a role in generating the upflows. However, it is worth noting that there are sections along these separatrix surfaces that do not show significant flows, either up or down. This does not imply that reconnection cannot cause upflows, because, for reconnection to occur, both an appropriate magnetic configuration and suitable plasma conditions (providing a region with low magnetic Reynolds number) are required. Such conditions may only be met for part, but not all, of a separatrix surface.

\subsection{Active Region NOAA 11419}

AR11419, located around $28^{\circ}$ north, crossed the disc between 12 February and 23 February, 2012 and was observed by EIS on 17 February at around 10:12 UT (Figure 3a-b). The sunspot complexity was deemed to be $\beta / \gamma$ at the start of the region's disc transit, but declined to $\alpha$ after 18 February. The number of spots peaked at six on 18 February. During disc passage there were only three C-class flares all of which occurred on 13 February. The region was not close to any $\mathrm{CH}$. It was more active during its previous rotation with several M-class flares and was observed by EIS at least 25 days after its emergence. The upflow speeds found around this region are lower than for the previous AR, with the strongest speeds to the south-west.

For this AR very little open field is found, according to the PFSS model, because the AR lies directly below a HCS null line and is almost entirely enclosed by its HCS curtains (Figure 4a). A small OFR lies to the south east of the AR 

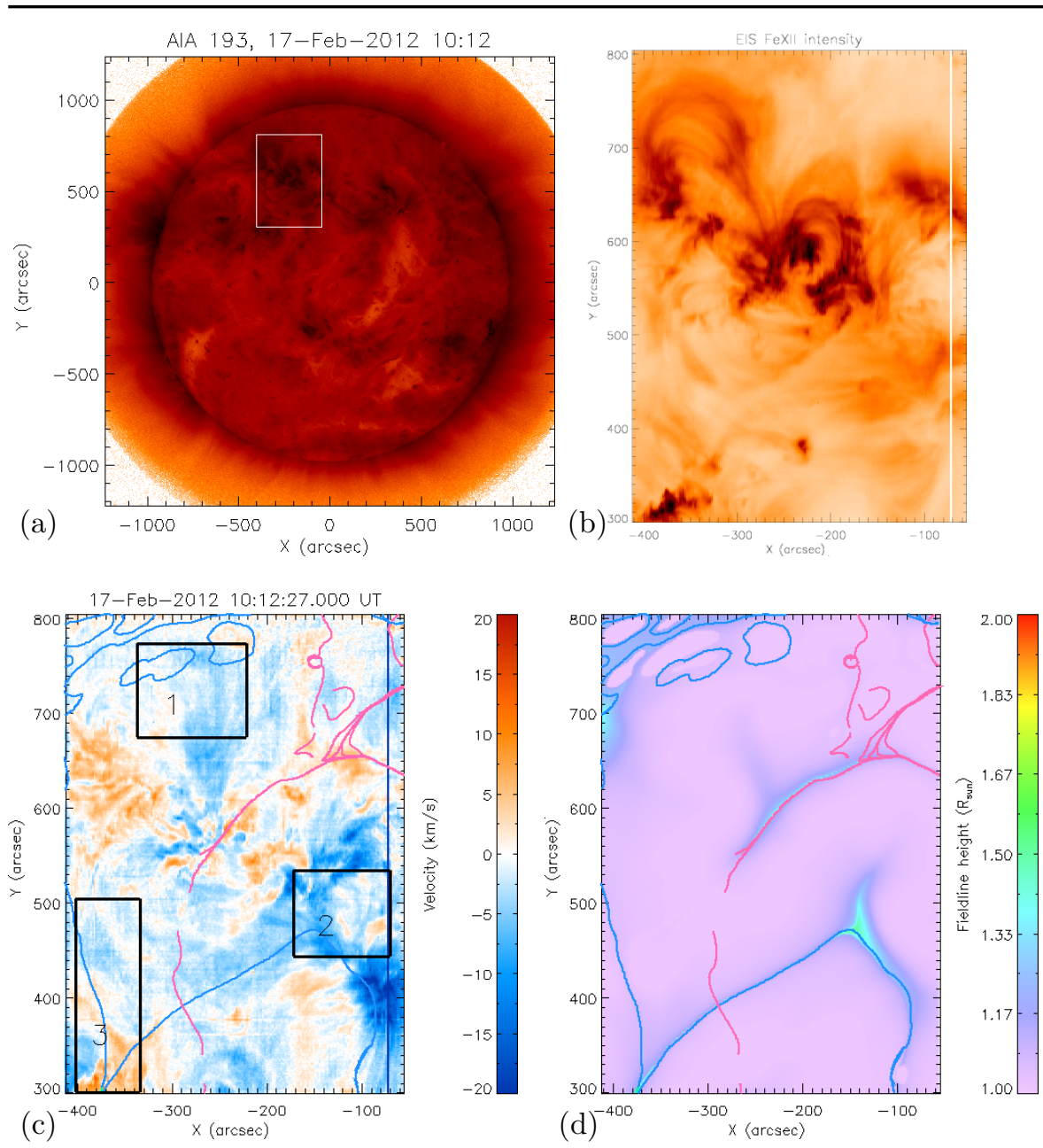

Figure 3. Same as for Figure 1, but showing AR 11419 on 17 February 2012.

corresponding to the open (yellow) field lines in Figure 4a, which does not overlap with the EIS raster. Therefore, none of the upflow regions observed in the EIS Doppler map (Figure 3c) are associated with open field. As in the previous case, we also consider the maximum heights reached by the closed field (Figure 3d) and find that the majority of field lines have maximum heights of less that 70 $\mathrm{Mm}\left(1.1 R_{\odot}\right)$. To the south west of the AR, where the strongest upflow is found, the field lines reach much higher, up to $420 \mathrm{Mm}$ (about $1.6 R_{\odot}$ ), suggesting that the upflows occur along extended (large) closed loops.

By tracing field lines from the photosphere (Figure 4b), we can see that there are long closed field lines that begin in the upflow regions of AR11419. These field lines diverge and connect, via either large high-reaching loops, to the negative polarity of the AR or, in long loops, to the negative polarity in the neighbouring 


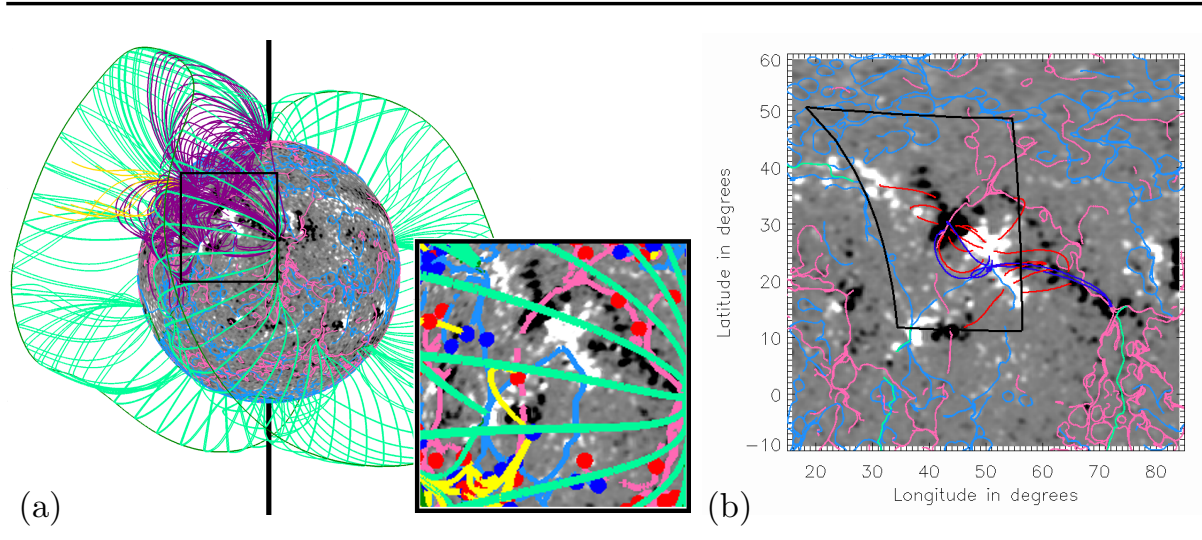

Figure 4. Same as for Figure 2, but showing AR 11419 as viewed at 10:12UT, 17 February 2012 .

$\mathrm{AR}$ on the west. Thus interchange reconnection between these regions may be a cause of the upflows.

The cut out from (Figure 4a) reveals that the blue separatrix surfaces that intersect the photosphere and thread the upflow regions come from a negative coronal null point and forms a dome. This dome is intersected by other separatrix surfaces associated with positive coronal null points and therefore there are two separators lying in this separatrix surface. These would make efficient sites for reconnection in the corona, which may cause the upflows.

It is interesting to note that elsewhere in the AR, the footprints of separatrix surfaces from positive coronal nulls are associated with downflows. For example, one such separatrix surface runs diagonally from $\left(-300^{\prime \prime}, 550^{\prime \prime}\right)$ to $\left(-100^{\prime \prime}, 650^{\prime \prime}\right)$ in Figure 3c. Figure 3d shows that this region is also associated with field lines reaching up to $\left(1.3 R_{\odot}\right) 210 \mathrm{Mm}$. The cutout from Figure 4 a does not show any separators associated with this separatrix surface and since the separatrix surface's footprint does not form part of a closed loop it cannot be a separatrix dome (see Platten et al. (2014) for more details on the different types of separatrix-surface structures).

\subsection{Active Region NOAA 11427}

Between 29 February and 6 March, 2012, AR11427 was located at about $15^{\circ}$ north, with some activity appearing to take place within the AR prior to 29 February. The AR was observed by EIS three days after its emergence, on 2 March with the raster starting at 07:25 UT. It was essentially bipolar throughout and had three spots. One C-flare occurred on 3 March and there were two C-class flares on 4 March. It was not close to any on-disc CHs.

In our PFSS extrapolation on 2 March 2012 the HCS null line forms two separate rings. Similar to the previous AR considered, the edge of one of these rings lies above the polarity inversion line associated with AR11427 and practically all the field within this AR lies within the HCS curtains (so is closed), although the 

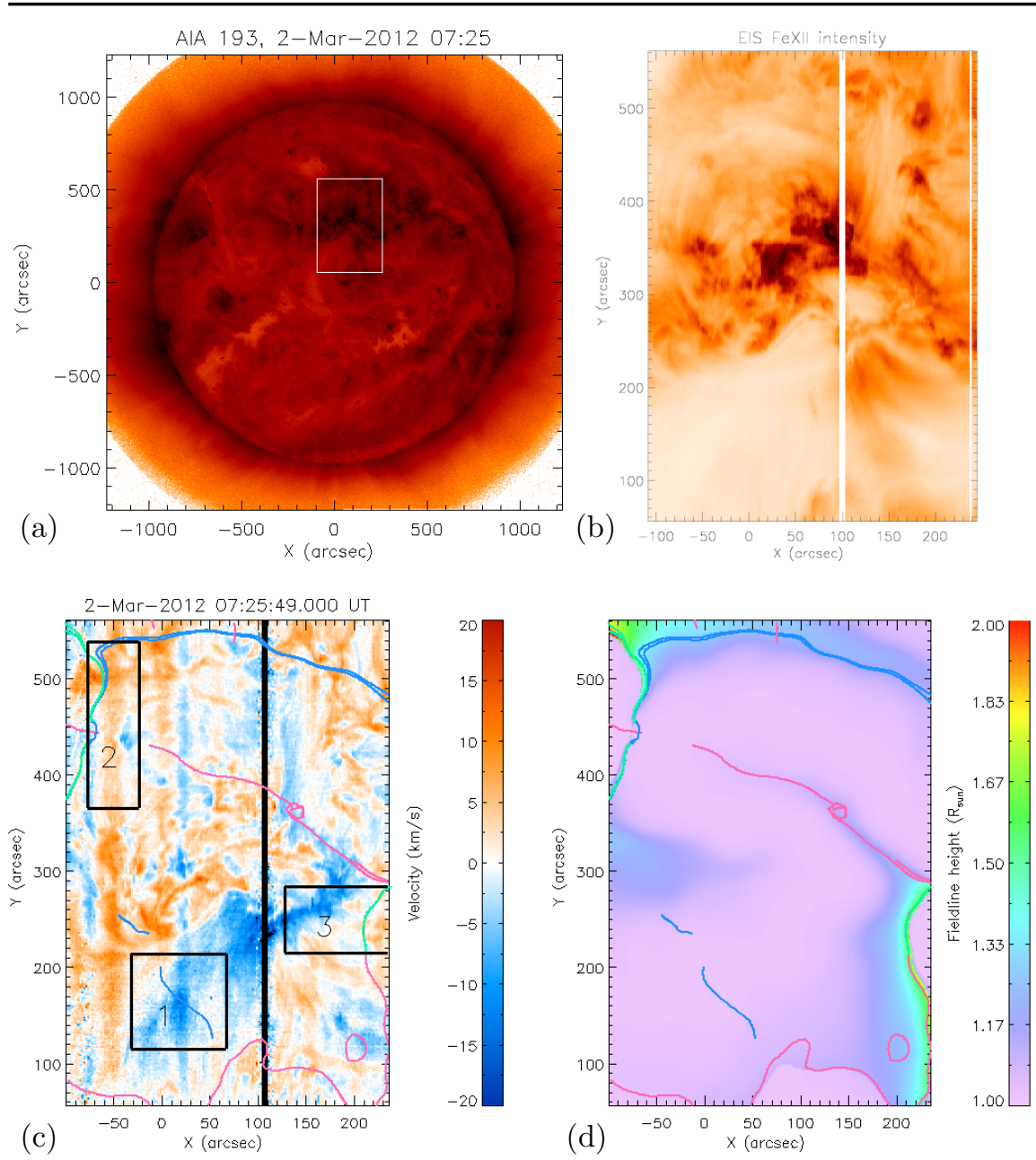

Figure 5. Same as for Figure 1, but showing AR 11427 on 2 March 2012.

edges of the AR, on either side, lie outside these HCS curtains. This means that field from AR11427 can form very large closed loops under the HCS curtain, as seen in the 3D plot of the coronal field at this time (Figure 6a). Only a few open field lines (yellow) are found and these emanate from the far edges of the AR (outwith the EIS field of view). The leading negative polarity is connected to the positive polarity via a set of large arching loops (wine lines) under the HCS curtain.

Comparing the upflows, observed in Fe XII (Figure 5c), with the intersection of the separatrix surfaces with the photosphere, we see that there is no obvious/consistent correspondence between these. Considering the cut out from Figure 6a there are no other topological features that are associated with the upflow regions. Also according to the model, any open field should lie to the north 

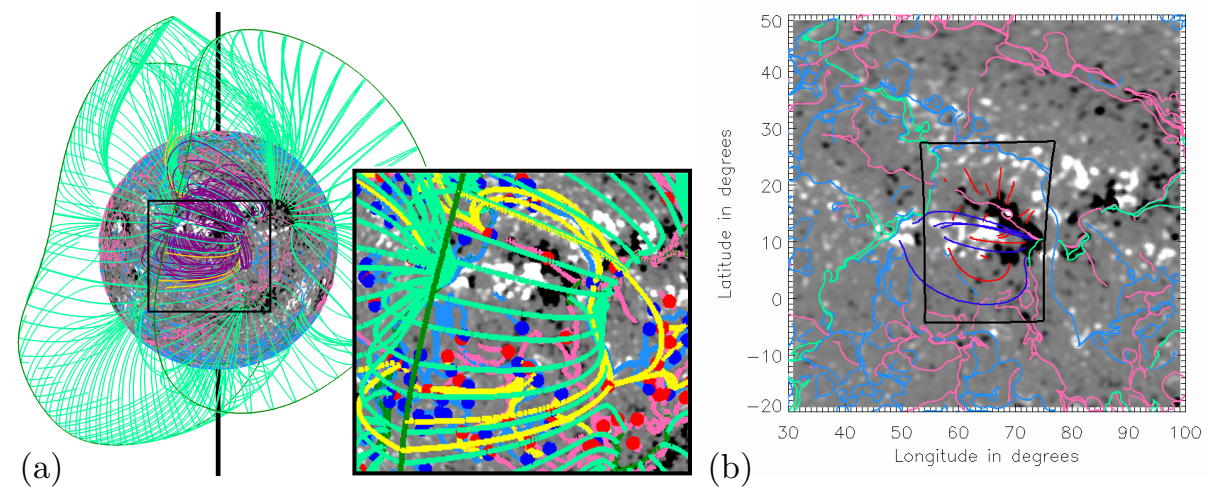

Figure 6. Same as for Figure 2, but showing AR 11427 as viewed at 7:25UT, 2 March 2012.

east or the south west of the EIS raster with foot points along the green lines shown in Figure 5d. These areas are not really associated with any upflowing material. Furthermore, all the upflow regions are associated with low-lying field lines (Figure 5d).

Field lines from the PFSS model anchored in the upflow regions, although low lying, are long (> $300 \mathrm{Mm}$ - blue line in Figure 6b) and arch across the AR. In reality these field lines may follow even longer, more complex paths, since, as discussed earlier, this AR shows flaring activity both before and shortly after 2nd March suggesting that our global potential field, which does not allow twisted structures, may be a poor representation of the true field.

\subsection{Active Region NOAA 11445}

The region, AR11445, located at about $23^{\circ}$ south, was identified on the disc between 23 March and 5 April 2012 when it crossed the west limb (Figure 7a). It was observed by EIS on 28 March at 10:20 UT (Figures 7b-c). Initially the region was magnetically complex $-\beta \gamma \delta$ on $23 / 24$ March, but simplified to $\beta$ from 25 March to 1 April and then became $\alpha$ on 2 through 5 April. A maximum spot count of 9 was reached on 30 March, after which the value fell to one. Flares occurred only on the first three days of disc transit: M 1.0 and C 6.5 flares occurred on 23 March, a C 7.2 flare on 24 March and a C 2.5 flare on 25 March. The region does not appear to be close to any on-disc CHs. It was first observed by the Solar TErrestrial RElations Observatory Behind spacecraft (STEREO-B; Kaiser et al., 2008) on 22 March, six days before the EIS observation. Figure 7c shows that the upflowing plasma is concentrated in small regions to the west and east of AR11445 with the strongest flow to the east.

Within this AR a small section of open field (yellow lines in Figure 8a) is seen to its north west. The other field lines (wine) traced from the AR and its surrounding area all appear to be closed with many arching high up into the solar atmosphere. 

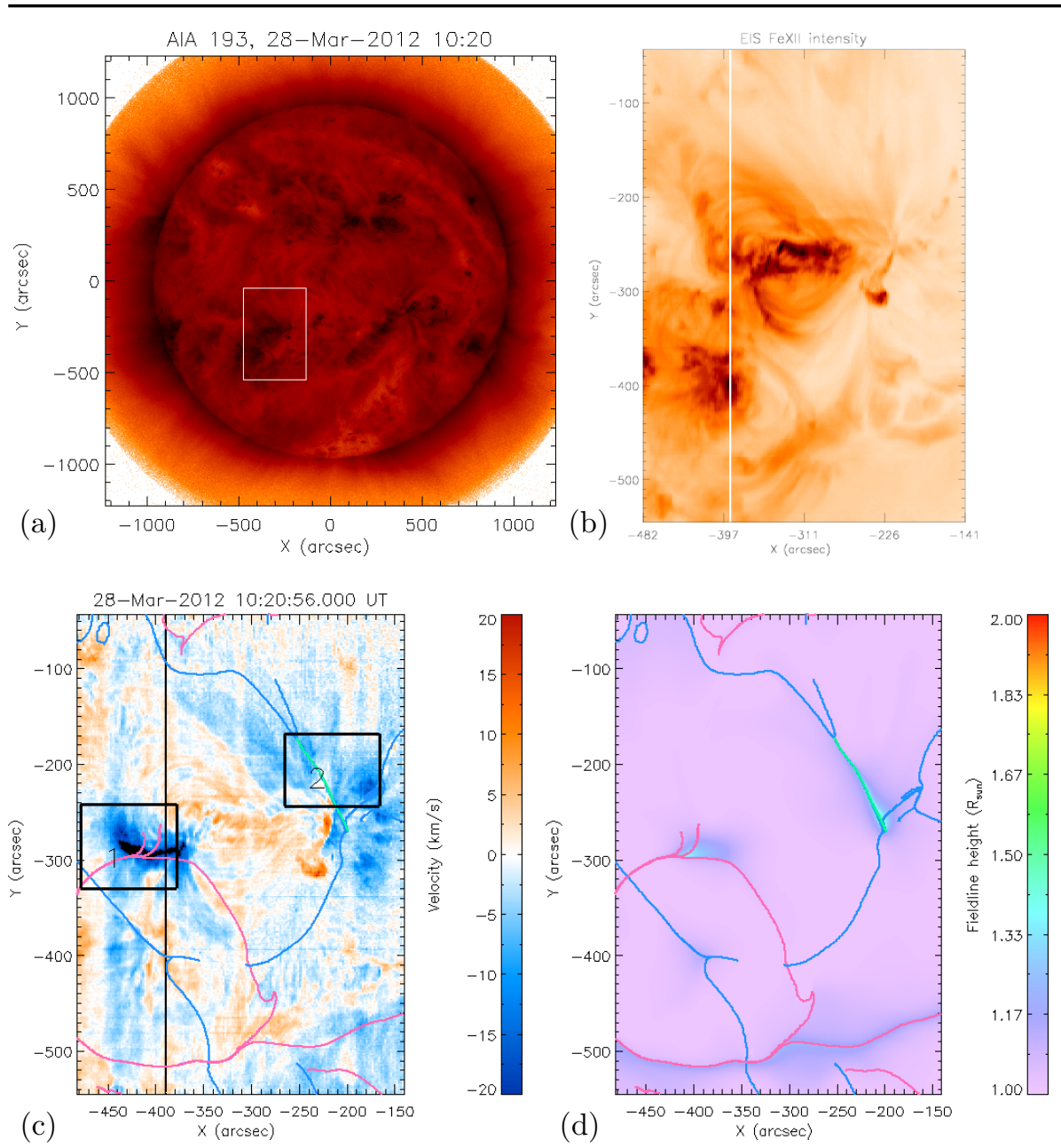

Figure 7. Same as for Figure 1, but showing AR 11445 on 28 March 2012.

When the intersection of the separatrix surfaces at the photospheric level are overlaid on the EIS velocity map (Figure 7c), we see that they cross the upflow regions. The strongest upflowing region is on the east side of the AR and is associated with separatrix surfaces from positive coronal null points, as well as a patch of fairly high reaching field ( $>250 \mathrm{Mm}$, Figure $7 \mathrm{~d}$ ). The upflowing region on the west, is associated with an extremely narrow OFR (i.e., the green line segment corresponding to the HCS curtain/photosphere intersection, to the west of the AR) surrounded by high reaching ( $>300 \mathrm{Mm})$ field.

Similarly, the long field lines (blue lines in Figure 8b) begin in locations where upflowing material is observed. Within the upflow region on the west of the AR there is not only a small amount of open field, but also evidence of a discontinuous mapping of field lines (blue field lines in Figure 8b) where high-arching field 

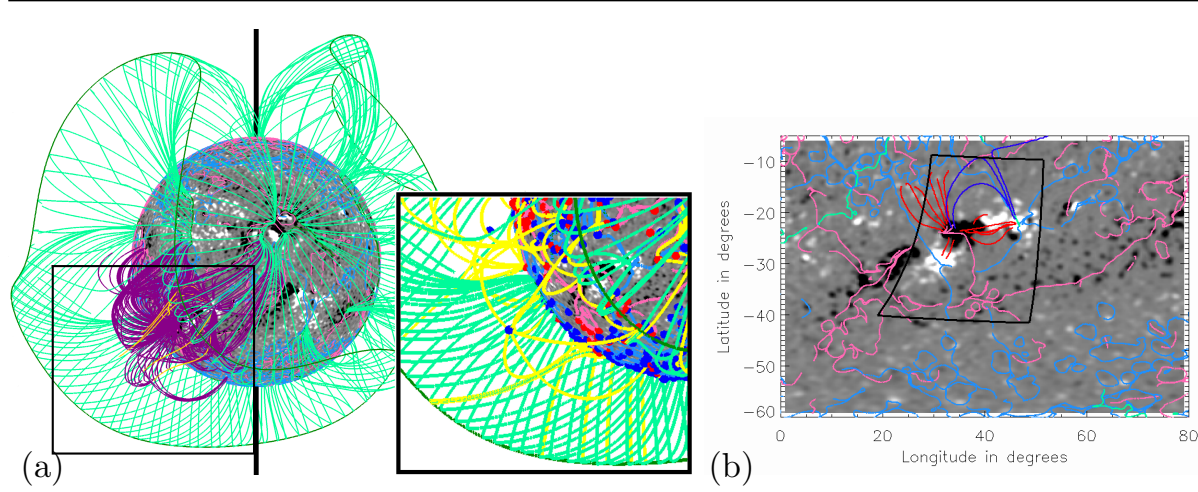

Figure 8. Same as for Figure 2, but showing AR 11445 as viewed at 10:20UT, 28 March 2012.

originating from this location connects to two very different areas on the solar surface. From the cut out from Figure 8a, we can see there is a negative coronal null point high above this AR whose separatrix surface intersects this western upflow region. This negative coronal null is connected to positive null points by five extended separators, which could be favourable reconnection sites. It is possible the separatrix surfaces from one or more of these positive nulls intersect the upflow region on the east of the AR and, thus, the upflows are associated with reconnection along one of these separators.

\subsection{Active Region NOAA 11553}

Active region 11553 was identified on the disc between 23 August and 4 September, 2012 (when it crossed the west limb) located at about $22^{\circ}$ south (Figure 9a). EIS observed it on 29 August at 3:10 UT (Figure 9b-c). The region was unipolar $(\alpha)$ with one spot during its first three days on disc. It became bi-polar $(\beta)$ from 26 August to 2 September and kept evolving reaching a maximum of nine spots. From $3-4$ September, it was $\beta \gamma$. No flares were produced throughout its disc passage and it was not close to any on-disc CHs. It was difficult to track this region due to missing STEREO-B data so we have not been able to estimate its age.

From Figure 9c, we see the upflowing plasma is concentrated to the north of the AR forming what appears to be loop like structures that arch from a region on the west where the fastest upflows are found. The EIS raster does not cover the most southern part of the AR, which may also show upflowing plasma.

In the PFSS model for 29 August 2012 (Figure 10a), the HCS null line has split into three separate loops: a sign that the magnetic fields from ARs completely dominate the bipolar field. Part of an HCS loop runs along the source surface directly above AR11553 with the associated HCS curtains enclosing most of the field from AR11553, except for its top northwestern region, close to where the strong upflows are found.

As can be seen from Figure 10a, there are many high-reaching closed field lines (wine coloured) extending from this AR. These lines are found furthest from the 

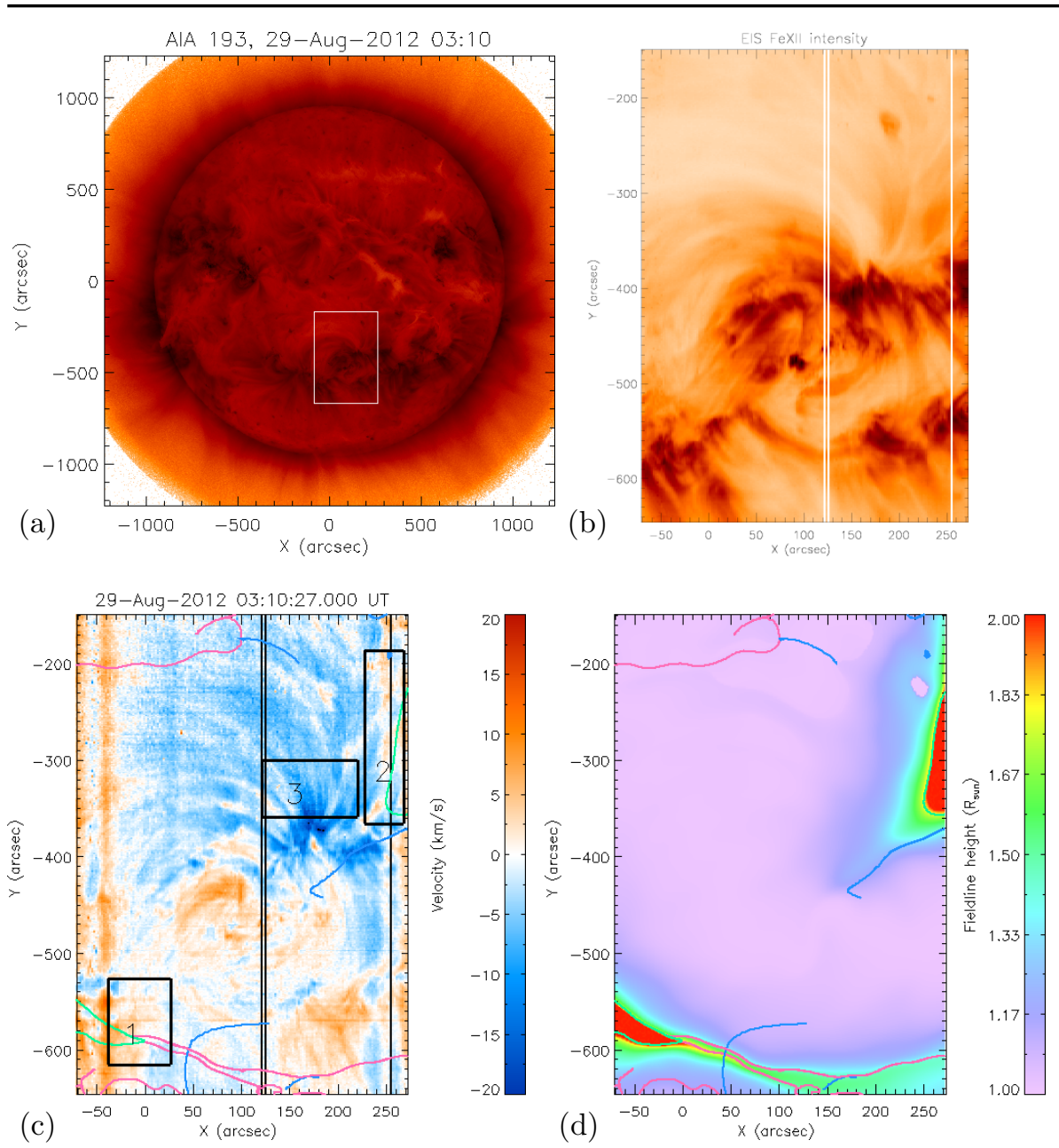

Figure 9. As for Fig. 1, but showing AR 11553 on 29 August 2012.

polarity inversion line through the AR, close to where the HCS curtains intersect with the photosphere. A positive-polarity OFR is found to the north west of the $\mathrm{AR}$ and a negative polarity OFR lies to its south east (yellow lines in Figure 10a). The EIS raster overlies the edge of each of these OFRs, as evidenced by the red patches in Figure 9d. The OFR on the top right edge of the EIS raster, and its associated extended region of high-reaching field $(>250 \mathrm{Mm})$, corresponds to where the upflows originate (c.f. Figure 9c): here interchange reconnection between open and closed field could cause the upflows. The second OFR, to the bottom left corner of the raster, corresponds to downflows rather than upflows.

By tracing sample field lines we can examine the connectivity of the system. Figure 10b shows that the longest field lines arch high over the AR (blue field lines) originating from near the northwest OFR and connecting to field out with 


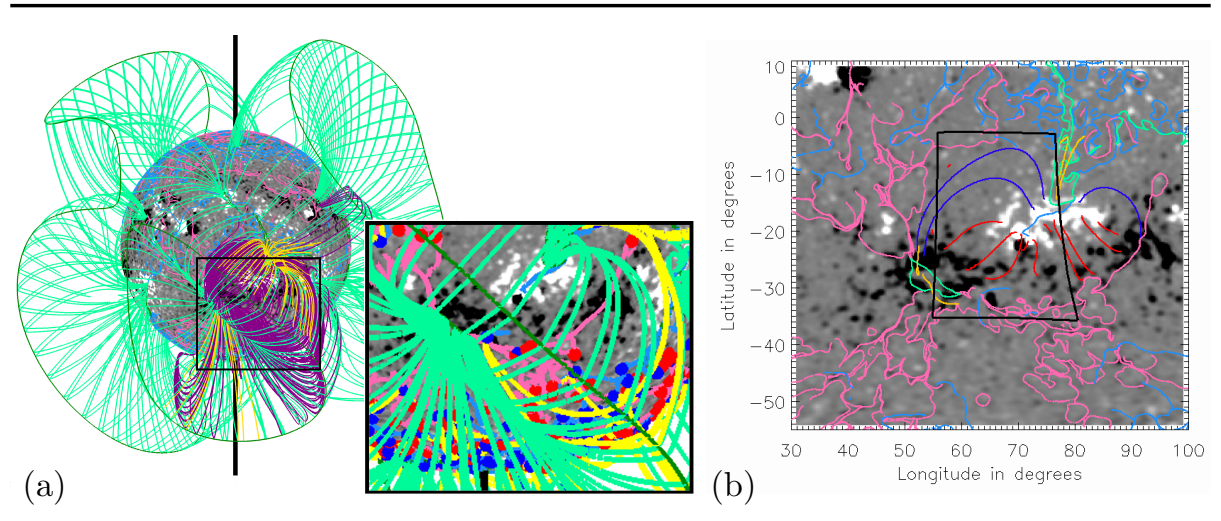

Figure 10. Same as for Figure 2, but showing AR 11553 as viewed at 3:10UT, 29 August 2012 .

the raster. From the bottom right of the raster, where the downflows are found, the field lines are much shorter and connect to field within the EIS raster. Sample open-field lines, traced in yellow, originate from the two OFRs, but none of these field lines cross the EIS raster field of view. The significant distortion of the EIS field-of-view when drawn on the synoptic map is caused by the high latitude location of the AR so projection effects could affect our interpretation of the AIA and EIS data.

When the intersection of the separatrix surfaces and HCS curtains with the photosphere are overlaid on the EIS Doppler velocity map (Figure 9c), we note that strongest regions of upflowing plasma (approximately $\left(200^{\prime \prime},-400^{\prime \prime}\right)$ ) reside close to the intersection of a separatrix surface with the photosphere, although not all of the upflow regions are threaded by separatrix surfaces. Looking at the topological features seen in the cut out of Figure 10a, there are no coronal null points directly above the AR or any separators arching over it. The negative null point associated with the separatrix surfaces that lie close to the strong upflows lies low in the corona and is visible in the cutout in Figure 10a. It has two separators connected to it and reconnection around these separators or between different closed-field regions separated by the separatrix surface may lead to the loops with upflowing plasma that EIS observed.

\subsection{Active Region NOAA AR11569}

Active region, AR11569, located at about $12^{\circ}$ south, was identified on the disc between 11 September and 22 September 2012 when it crossed the west limb (Figure 11a). It was observed by EIS on 15 September at 10:46 UT (Figure 11bc). The region's magnetic complexity increased from $\alpha / \gamma$ on 11 September to $\beta / \gamma$ on the next day. It remained at this level until 20 September when it reverted to $\alpha / \gamma$. The number of spots reached a maximum of 10 in the period 13 to 15 September after which it declined to one when the region left the disc. There were no flares produced throughout its disc passage. The region, located just to 
the north of a small on-disc $\mathrm{CH}$, was observed by EIS about 20 days after its emergence.
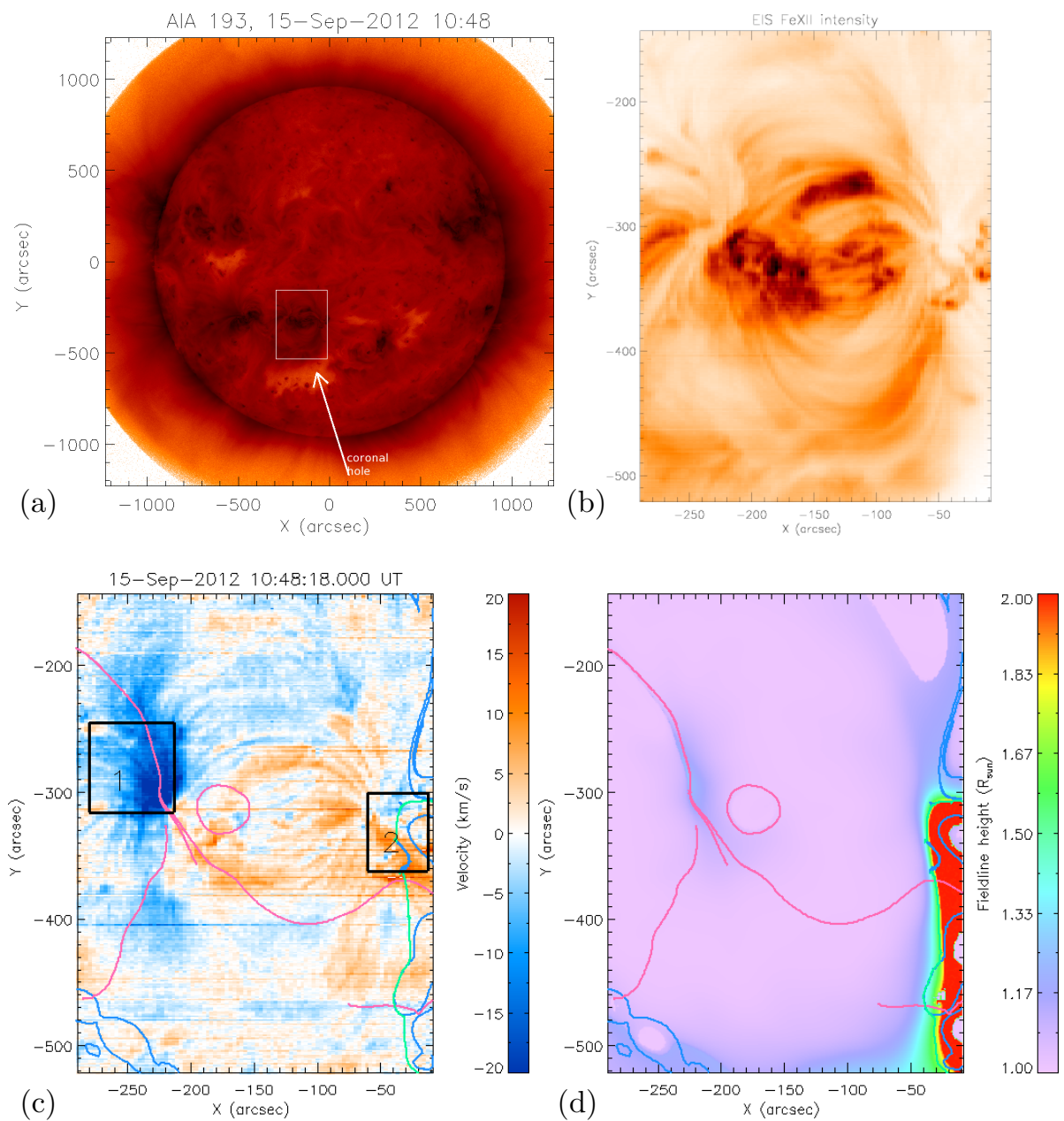

Figure 11. Same as for Figure 1, but showing AR 11569 on 15 September 2012.

Figure 11a shows that AR11569 lies to the south of the equator just west of another AR (AR11571). Strong upflowing plasma can be seen to the east of the AR whereas the plasma to the west of the AR appears to be flowing down (Figure 11c).

A 3D plot of the global potential field on 15 September 2012 (Figure 12a) shows that there is an OFR to the south west of the AR (yellow lines) which possibly corresponds to the on-disc $\mathrm{CH}$. From the AIA image it appears as if the EIS raster catches the edge of the $\mathrm{CH}$, in agreement with the OFR found in Figure 12a and the patch of red in Figure 11d. However, EIS only shows downflowing plasma in this part of the raster (Figure 11c) 


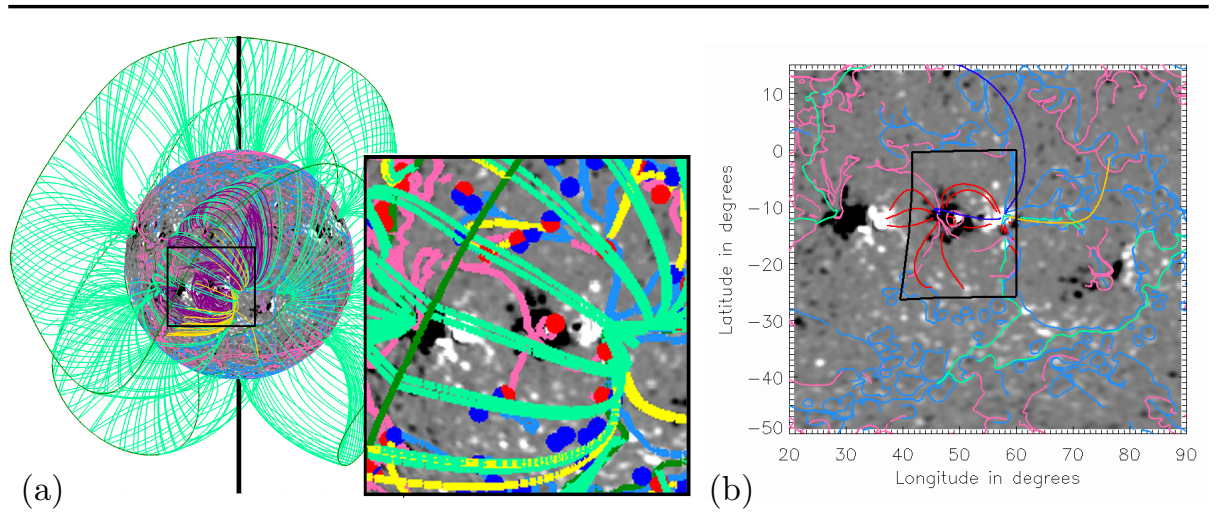

Figure 12. Same as for Figure 2, but showing AR 11569 as viewed at 10:48UT, 15 September 2012 .

The maximum heights reached by field lines from the region where strong upflows are observed (Figure $11 \mathrm{c}-\mathrm{d})$ reach high $\left(>230 \mathrm{Mm},>1.3 R_{\odot}\right)$ in the atmosphere. Figure 12a shows that this upflow region is clearly closed and lies comfortably under a pair of HCS curtains. However, a separatrix surface intersecting the photosphere is found running through this upflow region (Figure 11c) suggesting that reconnection may be a source of the upflows. The cut out from Figure 12a shows that no separators lie on this separatrix surface, which is associated with a positive coronal null and forms a wall-like structure.

Sample field lines from this AR are plotted in Figure $12 \mathrm{~b}$ over the synoptic magnetogram map along with the intersection of the positive/negative separatrix surfaces and HCS curtains with the photosphere. Field lines originating from the upflowing region are mostly short $(<300 \mathrm{Mm}$ in length; red) and either connect within the AR11569 itself or to the neighbouring AR11571. Only a few long field lines (> $300 \mathrm{Mm}$ in length; blue) are found originating from this region and linking to the western side of the AR.

\subsection{Active Region NOAA AR11575}

Located at about $8^{\circ}$ north, AR11575 was identified on the disc between 18 September and 1 October 2012 when it crossed the west limb (Figure 13a). It was observed by EIS on 23 September at 10:21UT (Figure 13b-c) when it was about fourteen days old. The region's magnetic structure was $\alpha \gamma$ from 18 September to 27 September. This complex region contained a maximum of 13 spots on 25 September. It was $\alpha \gamma$ and $\alpha$ during its remaining four days on the disc. Two C-class flares occurred on 25 September, with two more on 29 September, another on 30 September and a final one on 1 October. The region was not close to any on-disc CHs. From Figure 13c upflowing plasma is seen in and around the AR, but it is weak in most places except to the west of the region where strong compact upflows exist.

In Figure 14a, the HCS on the source surface is seen to form two separate loops. In this instance neither of the two HCS loops pass directly above the AR, 

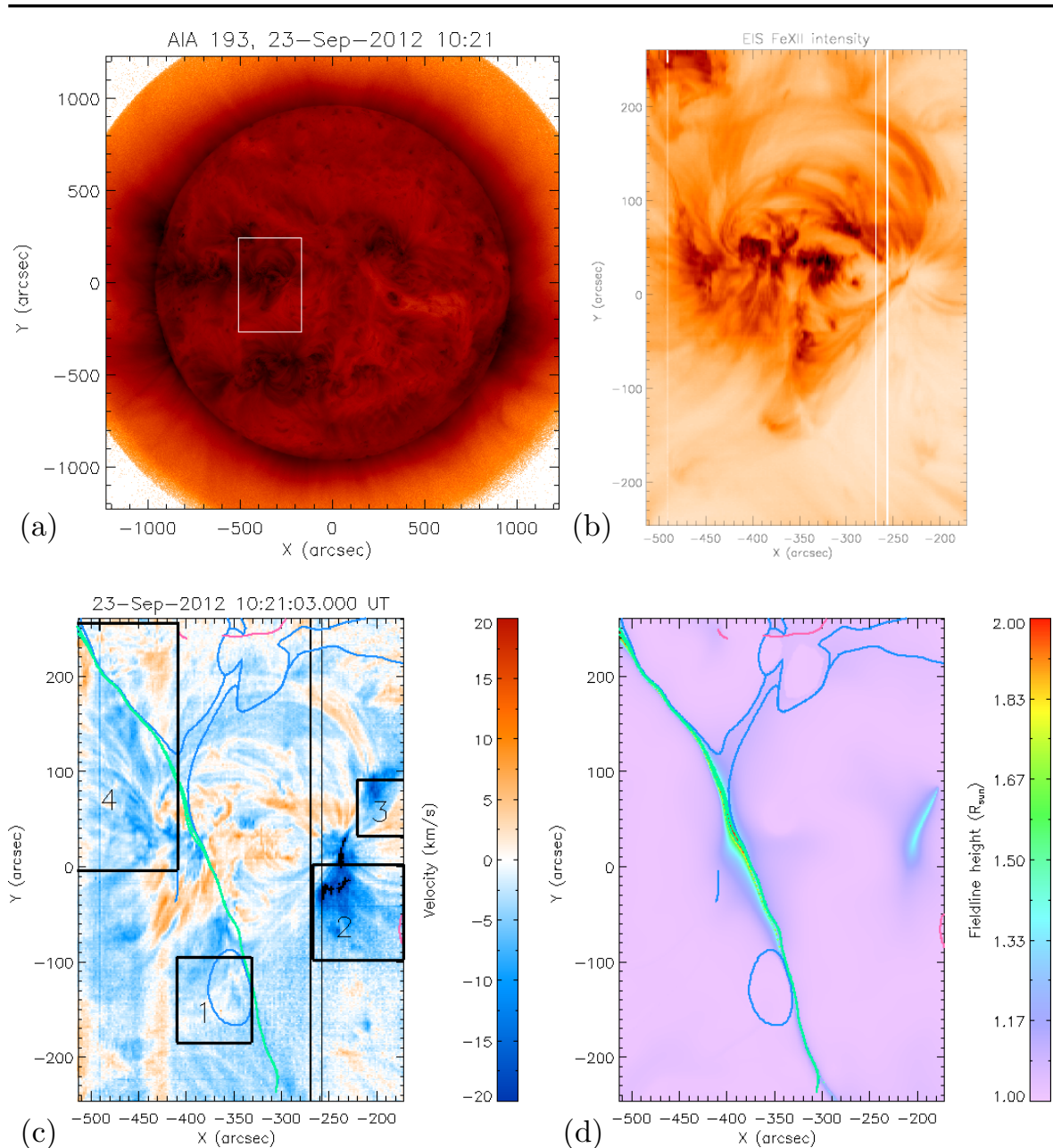

Figure 13. Same as for Figure 1, but showing AR 11575 on 23 September 2012.

instead they run overhead either side of the AR. Their HCS curtains run down to the photosphere, intersecting with it in a line through the positive sunspot of the AR, thus the central region of this sunspot is open (illustrated by yellow lines in Figure 14a). The closed field from this positive sunspot forms two distinct sets of field lines enclosed within the two separate sets of HCS curtains (wine lines in Figure 14a).

The HCS curtains, as predicted by the PFSS model, appear to lie a little to the west of where they should be in comparison to the loop structures seen in the EIS raster (Figures 13b-c). If the HCS curtains were moved slightly east then the loops illustrated in Figure 14a would agree with those observed by EIS.

Comparing the location of high-reaching field (Figure 13d) with the EIS Doppler map (Figure 13c), we find that the strongest blue-shifted regions on 

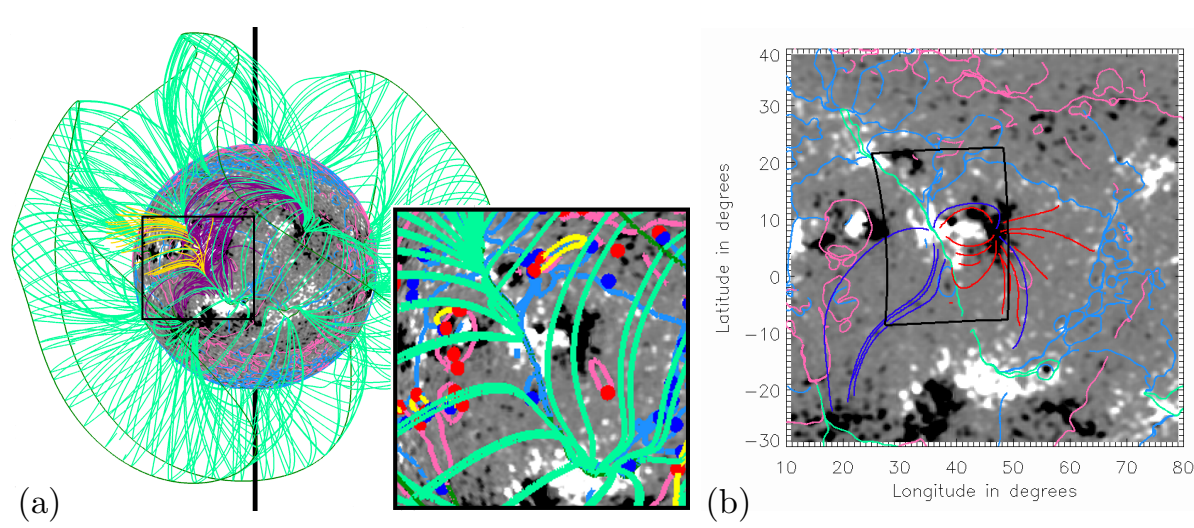

Figure 14. Same as for Figure 2, but showing AR 11575 as viewed at 10:21UT, 23 September 2012 .

the west of the AR are located very close to a patch of high-reaching (up to about $300 \mathrm{Mm}$ ) field lines. The OFR that runs through the centre of the positive sunspot, is also associated with an extended region of high reaching field (if the HCS curtains are shifted to the east). It is probable that the extended regions of weak loop-like upflows originate from either side of this OFR. The most likely cause of these extended upflowing loops emanating from the positive sunspot on the east of the AR appears to be interchange reconnection between open and closed field lines, but the origin of the strongest upflows from the negative spot on the west of the AR is unclear: there is no separarix surface intersecting the photosphere here.

To try and explain this we consider the topological features of this AR, as shown in the cut out of Figure $14 \mathrm{a}$, but there are no features of note in this area. Instead, from the long field lines traced from the negative pole of the AR (dark blue lines, Figure 14b), we see that some simply connect to the positive spot of the same AR, but others connect to far off positive regions to the south and further west. Reconnection between these field lines could cause the strong upflows observed about the negative spot of this AR.

\section{Results}

Before we discuss the results, we highlight the limitations of the analysis carried out. Firstly, the potential field nature of the PFSS model will not perfectly replicate the true field structure around the ARs, but the model should give a reasonable guide to how the global/large-scale fields from the ARs connect to their surroundings. Furthermore, due to the resolution of the HMI synoptic data, the number of harmonics used to perform the extrapolations was constrained (though this number was much higher than for any previous PFSS model). This means that the coronal null points and associated separatrix surfaces found in 
the PFSS are only a subset of all the coronal nulls and separatrix surfaces that exist. In reality there would be many more coronal nulls, most of which would be comparatively low lying, although their separatrix surfaces and any associated separators may still have global consequences (Edwards, 2014).

Secondly, the EIS measurements are taken through optically thin plasma and projection effects might occur. These are mitigated as much as they can be by choosing ARs close to disc centre, thus minimising the distortions from integrating in the line of sight, but there may be a shift when the EIS raster is aligned with features at the photospheric level (for example, the HMI magnetic field or the separatrix-surface intersections with the photosphere). Despite the limitations of both the coronal measurements and the PFSS model, we find reasonable agreement between the observed and predicted loop structures and also find trends in the observed upflows.

As may be seen from Table 1, the upflows rarely correspond to OFRs and so, in the majority of cases, they are not contributing to solar wind. The patches of upflow mainly correspond to the locations where separatrix surfaces intercept the photosphere. In the cases where this is not true it is usually because the observed upflows are associated with loops. However, by following these loops to where they originate, we typically find evidence of a separatrix surface footprint. From the PFSS model, we find that in many cases the magnetic field associated with upflows forms large loops that follow the separatrix surfaces and often extend around the top of large separatrix domes. A second key ingredient for upflows appears to be high-reaching (up to and above $250 \mathrm{Mm}$ ) field lines. Indeed, a good correspondence exists between the locations of the upflows and the maximum field line heights. Furthermore, the locations of the separatrix surfaces at the photosphere mostly correspond to the locations of high-reaching (around 250 $\mathrm{Mm}$ ) field lines.

In Table 1 , we detail the plasma temperature $(T)$, density $\left(n_{\mathrm{e}}\right)$ and emission measure (EM) and also the FIP bias measured within the black boxes outlined in the earlier figures (called Doppler regions in the table). These regions over lap areas of upflow that appear to be either at the base of loops or part way along loop structures, as well as regions with up and down flowing plasma. The temperatures of these regions vary between 1.6 MK to above $4 \mathrm{MK}$ (which is the highest temperature constraint we have using Fe XVII), the densities lie between $2.7 \times 10^{8} \mathrm{~cm}^{-3}$ to $1.5 \times 10^{9} \mathrm{~cm}^{-3}$ and the emission measures are between $1.8 \times 10^{27} \mathrm{~cm}^{-5}$ to $1.8 \times 10^{28} \mathrm{~cm}^{-5}$. However, there appear to be no standout characteristics that allow regions of strong flow to be distinguished from regions of weak up flow or mixed (up and down) flow. Similarly, there are no significant differences in these plasma parameters between regions of open and closed field.

Regarding the FIP bias in each AR, there is again no clear trend in these values with respect to the direction of the flows in the region or whether the field is open or closed. For example, the strongest upflow in AR1569 has a FIP bias of 2.4 compared to 2.2 in the downflowing plasma and the strongest upflow in AR11445 has a FIP bias of 2.2 compared to 1.9 in a weakly upflowing region. In AR11553, the FIP bias in box 3, which overlies the strongest upflows, is similar to the FIP bias in box 2, which contains much weaker upflows, but a much higher FIP bias is found in box 1 which contains downflowing plasma. 
In the case of AR11427, the two highest FIP bias values are found in boxes 2 and 3 which contain narrow OFRs, similarly the two boxes overlying OFRs in AR11553 have highest FIP bias value. On the other hand, highest FIP bias in AR11271 is not associated with the box located in the OFR. It should be noted, however, that the errors on the FIP bias are of the order of $30 \%$ and therefore in some ARs the values are too close to be sure.

In terms of vicinity to observed CHs, AR11271 and AR11569 had CHs nearby that were visible in AIA $193 \AA$. In both of these cases there was evidence of open magnetic field from the PFSS model. However, the PFSS models also found open fields associated with other ARs. In three of these cases (AR11427, AR11445 and AR11575), the photospheric footprint of the open field was extremely narrow and in the fourth, AR11553, the open-field footprint was small, therefore it is not surprising that these did not produce signatures of $\mathrm{CHs}$ that could be observed in the AIA $193 \AA$ images.

From the relatively simple analysis tools and diagnostics we have tested, we find no firm identifications of locations of slow solar wind sources, but there are a few comments that can be made about the different types of observed upflow regions.

1. Strong compact sources of upflow that appear to be the base of large loops are typically associated with the following:

a) a higher FIP bias value than is found in the surrounding AR.

b) regions of high-reaching $(250 \mathrm{Mm})$, but not necessarily open field

c) the photospheric footprint of a separatrix surface

2. For upflow regions that are associated with loop-like structures:

a) FIP bias values may be high or low.

b) At the possible origin of these loops (which may or may not be in the boxed region containing the loops), there is usually evidence of a separatrix surface footprint and/or open/high-reaching field.

3. For regions of weak upflows with no or an indistinct loop-like signature

a) FIP bias values are not as high as for the strong blue-shifted regions in the area

b) There are not necessarily any corresponding features such as separatrix surface footprints or high-reaching fields.

Thus both high-reaching field, or open field, and the existence of a separatrix surface footprint seem to be the most common features associated with upflows.

\section{Discussion}

The principal aim of this work was to assess whether or not the recently discovered active-region upflows can in general access open magnetic field and ultimately contribute to the slow solar wind. Previous work, summarised in the introduction, focused on a pair of well-studied AR complexes that were visible 
on the Sun in December 2007 and January 2008. These very detailed studies did establish, for both cases, a connection to the solar wind that was verified, later, by the detection of active-region-composition plasma by the ACE spacecraft. In the present study of seven ARs, selected from a wide range of solar latitudes, we have not yet attempted any comparison with ACE observations. Instead, we have sought to relate, for all seven ARs, the observed plasma upflows and the plasma FIP-bias to regions of open magnetic field as determined by PFSS models from HMI daily synoptic data (field is "open" if it reaches the source surface which is set at $2.0 R_{\odot}$ ).

Using the Doppler velocity data determined from EIS Fe XII, we can see that all of the ARs considered demonstrate upflows on at least one side. When each region is compared with its global potential field source surface model only one AR (AR11271 observed on 21 August 2011) shows a large OFR aligned with one of the upflow regions. In addition, three regions (AR11427 on 2 March 2012, AR11445 on 28 March 2012, and AR11575 on 23 September 2012) have narrow channels of open field that are close to upflows and so could be associated with them.

On 17 February the AR (AR11419) within the EIS field of view contains only closed magnetic field according to the PFSS extrapolation, but the upflows observed in this AR line up with the intersection of the separatrix surfaces with the lower boundary. This is in agreement with the result of Baker et al. (2009), who found that upflows occur in the presence of quasi-separatrix layers (QSLs).

The AR observed on 29 August 2012 (AR11553) had OFRs on either side. One of these regions lies close to a strong compact region of upflows, the other overlies a region of downflow. Due to the southerly location of this AR it is possible that projection effects are important. However, it should also be noted that, around the time of observation, this AR had flux emergence to the south and the polarities of the AR changed quite dramatically. Since the synoptic magnetogram maps are produced from a 4-h average on the near side of the Sun, and a Carrington rotation average on the far side, if there has been significant flux emergence on the far side of the Sun then they will not accurately represent the magnetic field in the photosphere.

Finally for 15 September AR11569, there is some open field associated with the sample box to the west of the region. Here, the velocity measurements show a predominance of downflows whilst FIP-bias values are the largest observed. The origin of these downflows is not clear, but there are small-scale loops in this location with which they could be associated.

Overall, even with the simplicity of a potential field extrapolation, we have shown that OFRs (which are often very small) can, but do not always, occur within regions where upflows are observed. Furthermore, usually only one side of an AR will have some open field, although this is not always the case, as was seen, for example, in AR11553 on 29 August 2012. Thus, regions of upflow, which are frequently found on both side of an AR, are not necessarily direct sources of the slow solar wind.

There appears to be a strong association between the upflow regions, separatrix surfaces and high-reaching field lines. Baker et al. (2009) suggested that upflows are initially driven by reconnection between comparatively small AR 
loops and much larger structures. Our results broadly agree with this, however, in our study, the large-scale structures are more often than not closed, rather than open. Furthermore, we find evidence that the reconnection could also be between two large-scale topologically distinct regions containing high-reaching and/or extended field lines.

There does not seem to be a clear link between the highest FIP-bias values and the derived position of open field from the PFSS model. On average the FIP-bias measurements from AR11419 on 17 February 2012 are lower than those from the other six ARs all of which we predict have some open field (ignoring the measurements from 29 August 2012 where we do not have an accurate prediction about the open field). However, we do not have a large enough sample of data to say for certain if this is a trend. Nevertheless, the range of observed FIP-bias values, which is 1.6 to 3.7 , is such that the plasma would be recognized as being of slow solar wind origin were it later to be detected by ACE.

Finally, although all of the ARs studied have one or more associated plasma upflows, only one of these - AR 11271 on 21 August 2011 - shows a region of upflow that is aligned with an extended region of open magnetic field. This upflow region is therefore a likely contributor to the slow solar wind. For the remaining six ARs there is no evidence of a direct contribution to the slow wind. However the extended magnetic structures, which contain the upflowing material, may, through further reconnections at separators or separatrix curtains, be able to transfer this plasma to remote open field structures (for an example, see Culhane et al., 2014). The identification of such situations would require further detailed magnetic field modelling which is beyond the scope of the present paper.

Acknowledgments SJE acknowledges the financial support of the Isle of Man Government during her $\mathrm{PhD}$ and the support of the STFC. CEP acknowledges the support of the St Andrews SMTG's STFC consolidated grant. The work of DHB was performed under contract with the Naval Research Laboratory and was funded by the NASA Hinode program. Hinode is a Japanese mission developed and launched by ISAS/JAXA, collaborating with NAOJ as a domestic partner, NASA and STFC (UK) as international partners. The Hinode science team organized at ISAS/JAXA conducts the scientific operation of Hinode mission: this team mainly consists of scientists from institutes in the partner countries. Support for the post-launch operation is provided by JAXA and NAOJ (Japan), STFC (U.K.), NASA, ESA, and NSC (Norway). Courtesy of NASA/SDO and the AIA, EVE, and HMI science teams. The research leading to these results has received funding from the European Commission's Seventh Framework Programme under the grant agreement No. 284461 (eHEROES project).

\section{References}

Baker, D., van Driel-Gesztelyi, L., Mandrini, C.H., Démoulin, P., Murray, M.J.: 2009, Magnetic reconnection along quasi-separatrix layers as a driver of ubiquitous active region outflows. Astrophys. J. 705, 926. DOI. ADS. 
Brooks, D.H., Warren, H.P.: 2011, Establishing a connection between active region outflows and the solar wind: Abundance measurements with EIS/Hinode. Astrophys. J. Lett. 727, L13. DOI. ADS

Brooks, D.H., Warren, H.P.: 2012, The coronal source of extreme-ultraviolet line profile asymmetries in solar active region outflows. Astrophys. J. Lett. 760, L5. DOI. ADS.

Bryans, P., Young, P.R., Doschek, G.A.: 2010, Multiple component outflows in an active region observed with the EUV Imaging Spectrometer on Hinode. Astrophys. J. 715, 1012. DOI. ADS.

Culhane, J.L., Harra, L.K., James, A.M., Al-Janabi, K., Bradley, L.J., Chaudry, R.A., Rees, K., Tandy, J.A., Thomas, P., Whillock, M.C.R., Winter, B., Doschek, G.A., Korendyke, C.M., Brown, C.M., Myers, S., Mariska, J., Seely, J., Lang, J., Kent, B.J., Shaughnessy, B.M., Young, P.R., Simnett, G.M., Castelli, C.M., Mahmoud, S., Mapson-Menard, H., Probyn, B.J., Thomas, R.J., Davila, J., Dere, K., Windt, D., Shea, J., Hagood, R., Moye, R., Hara, H., Watanabe, T., Matsuzaki, K., Kosugi, T., Hansteen, V., Wikstol, Ø.: 2007, The EUV Imaging Spectrometer for Hinode. Solar Phys. 243, 19. DOI. ADS.

Culhane, J.L., Brooks, D.H., van Driel-Gesztelyi, L., Démoulin, P., Baker, D., DeRosa, M.L., Mandrini, C.H., Zhao, L., Zurbuchen, T.H.: 2014, Tracking solar active region outflow plasma from its source to the near-Earth environment. Solar Phys. 289, 3799. DOI. ADS.

De Pontieu, B., McIntosh, S.W., Hansteen, V.H., Schrijver, C.J.: 2009, Observing the roots of solar coronal heating - in the chromosphere. Astrophys. J. Lett. 701, L1. DOI. ADS.

Del Zanna, G., Aulanier, G., Klein, K.-L., Török, T.: 2011, A single picture for solar coronal outflows and radio noise storms. Astron. Astrophys. 526, A137. DOI. ADS.

Dere, K.P., Landi, E., Mason, H.E., Monsignori Fossi, B.C., Young, P.R.: 1997, CHIANTI An atomic database for emission lines. Astron. Astrophys. Suppl. 125, 149. DOI. ADS.

Doschek, G.A., Warren, H.P., Mariska, J.T., Muglach, K., Culhane, J.L., Hara, H., Watanabe, T.: 2008, Flows and nonthermal velocities in solar active regions observed with the EUV Imaging Spectrometer on Hinode: A tracer of active region sources of heliospheric magnetic fields? Astrophys. J. 686, 1362. DOI. ADS.

Edwards, S.J.: 2014, On the topology of global coronal magnetic fields. Ph.D. thesis, University of St Andrews.

Grevesse, N., Asplund, M., Sauval, A.J.: 2007, The solar chemical composition. Space Sci. Rev. 130, 105. DOI. ADS.

Handy, B.N., Acton, L.W., Kankelborg, C.C., Wolfson, C.J., Akin, D.J., Bruner, M.E., Caravalho, R., Catura, R.C., Chevalier, R., Duncan, D.W., Edwards, C.G., Feinstein, C.N., Freeland, S.L., Friedlaender, F.M., Hoffmann, C.H., Hurlburt, N.E., Jurcevich, B.K., Katz, N.L., Kelly, G.A., Lemen, J.R., Levay, M., Lindgren, R.W., Mathur, D.P., Meyer, S.B., Morrison, S.J., Morrison, M.D., Nightingale, R.W., Pope, T.P., Rehse, R.A., Schrijver, C.J., Shine, R.A., Shing, L., Strong, K.T., Tarbell, T.D., Title, A.M., Torgerson, D.D., Golub, L., Bookbinder, J.A., Caldwell, D., Cheimets, P.N., Davis, W.N., Deluca, E.E., McMullen, R.A., Warren, H.P., Amato, D., Fisher, R., Maldonado, H., Parkinson, C.: 1999, The Transition Region And Coronal Explorer. Solar Phys. 187, 229. DOI. ADS.

Hara, H., Watanabe, T., Harra, L.K., Culhane, J.L., Young, P.R., Mariska, J.T., Doschek, G.A.: 2008, Coronal plasma motions near footpoints of active region loops revealed from spectroscopic observations with Hinode EIS. Astrophys. J. Lett. 678, L67. DOI. ADS.

Harra, L.K., Sakao, T., Mandrini, C.H., Hara, H., Imada, S., Young, P.R., van Driel-Gesztelyi, L., Baker, D.: 2008, Outflows at the edges of active regions: Contribution to solar wind formation? Astrophys. J. Lett. 676, L147. DOI. ADS.

Harra, L.K., Archontis, V., Pedram, E., Hood, A.W., Shelton, D.L., van Driel-Gesztelyi, L.: 2012, The creation of outflowing plasma in the corona at emerging flux regions: Comparing observations and simulations. Solar Phys. 278, 47. DOI. ADS.

Haynes, A.L., Parnell, C.E.: 2007, A trilinear method for finding null points in a threedimensional vector space. Phys. Plasmas 14, 082107. DOI. ADS

Haynes, A.L., Parnell, C.E.: 2010, A method for finding three-dimensional magnetic skeletons. Phys. Plasmas 17, 092903. DOI. ADS

He, J.-S., Marsch, E., Tu, C.-Y., Guo, L.-J., Tian, H.: 2010, Intermittent outflows at the edge of an active region - A possible source of the solar wind? Astron. Astrophys. 516, A14. DOI. ADS.

Kaiser, M.L., Kucera, T.A., Davila, J.M., St. Cyr, O.C., Guhathakurta, M., Christian, E.: 2008, The STEREO mission: An introduction. Space Sci. Rev. 136, 5. DOI. ADS. 
Kashyap, V., Drake, J.J.: 1998, Markov-chain Monte Carlo reconstruction of emission measure distributions: Application to solar extreme-ultraviolet spectra. Astrophys. J. 503, 450. DOI. ADS.

Kashyap, V., Drake, J.J.: 2000, PINTofALE : Package for the interactive analysis of line emission. Bull. Astron. Soc. India 28, 475. ADS.

Kosugi, T., Matsuzaki, K., Sakao, T., Shimizu, T., Sone, Y., Tachikawa, S., Hashimoto, T., Minesugi, K., Ohnishi, A., Yamada, T., Tsuneta, S., Hara, H., Ichimoto, K., Suematsu, Y., Shimojo, M., Watanabe, T., Shimada, S., Davis, J.M., Hill, L.D., Owens, J.K., Title, A.M., Culhane, J.L., Harra, L.K., Doschek, G.A., Golub, L.: 2007, The Hinode (Solar-B) mission: An overview. Solar Phys. 243, 3. DOI. ADS.

Laming, M.: 2014 Living Rev. Solar Phys., submitted.

Landi, E., Del Zanna, G., Young, P.R., Dere, K.P., Mason, H.E.: 2012, CHIANTI - An atomic database for emission lines. XII. Version 7 of the database. Astrophys. J. 744, 99. DOI. ADS.

Lemen, J.R., Title, A.M., Akin, D.J., Boerner, P.F., Chou, C., Drake, J.F., Duncan, D.W., Edwards, C.G., Friedlaender, F.M., Heyman, G.F., Hurlburt, N.E., Katz, N.L., Kushner, G.D., Levay, M., Lindgren, R.W., Mathur, D.P., McFeaters, E.L., Mitchell, S., Rehse, R.A., Schrijver, C.J., Springer, L.A., Stern, R.A., Tarbell, T.D., Wuelser, J.-P., Wolfson, C.J., Yanari, C., Bookbinder, J.A., Cheimets, P.N., Caldwell, D., Deluca, E.E., Gates, R., Golub, L., Park, S., Podgorski, W.A., Bush, R.I., Scherrer, P.H., Gummin, M.A., Smith, P., Auker, G., Jerram, P., Pool, P., Soufli, R., Windt, D.L., Beardsley, S., Clapp, M., Lang, J., Waltham, N.: 2012, The Atmospheric Imaging Assembly (AIA) on the Solar Dynamics Observatory (SDO). Solar Phys. 275, 17. DOI. ADS.

Mackay, D., Yeates, A.: 2012, The sun's global photospheric and coronal magnetic fields: Observations and models. Living Rev. Solar Phys. 9, (6). DOI. ADS.

Mandrini, C.H., Schmieder, B., Démoulin, P., Guo, Y., Cristiani, G.D.: 2014, Topological analysis of emerging bipole clusters producing violent solar events. Solar Phys. 289, 2041. DOI. ADS.

Parnell, C.E., Smith, J.M., Neukirch, T., Priest, E.R.: 1996, The structure of three-dimensional magnetic neutral points. Phys. Plasmas 3, 759. DOI. ADS.

Platten, S.J., Parnell, C.E., Haynes, A.L., Priest, E.R., Mackay, D.H.: 2014, The solar cycle variation of topological structures in the global solar corona. Astron. Astrophys. 565, A44. DOI. ADS.

Sakao, T., Kano, R., Narukage, N., Kotoku, J., Bando, T., DeLuca, E.E., Lundquist, L.L., Tsuneta, S., Harra, L.K., Katsukawa, Y., Kubo, M., Hara, H., Matsuzaki, K., Shimojo, M., Bookbinder, J.A., Golub, L., Korreck, K.E., Su, Y., Shibasaki, K., Shimizu, T., Nakatani, I.: 2007, Continuous plasma outflows from the edge of a solar active region as a possible source of solar wind. Science $\mathbf{3 1 8}$. DOI. ADS

Scherrer, P.H., Schou, J., Bush, R.I., Kosovichev, A.G., Bogart, R.S., Hoeksema, J.T., Liu, Y., Duvall, T.L., Zhao, J., Title, A.M., Schrijver, C.J., Tarbell, T.D., Tomczyk, S.: 2012, The Helioseismic and Magnetic Imager (HMI) investigation for the Solar Dynamics Observatory (SDO). Solar Phys. 275, 207. DOI. ADS.

Slemzin, V., Harra, L., Urnov, A., Kuzin, S., Goryaev, F., Berghmans, D.: 2013, Signatures of slow solar wind streams from active regions in the inner corona. Solar Phys. 286, 157. DOI. ADS.

Ugarte-Urra, I., Warren, H.P.: 2011, Temporal variability of active region outflows. Astrophys. J. 730, 37. DOI. ADS.

van Ballegooijen, A.A., Cartledge, N.P., Priest, E.R.: 1998, Magnetic flux transport and the formation of filament channels on the Sun. Astrophys. J. 501, 866. DOI. ADS.

van Driel-Gesztelyi, L., Culhane, J.L., Baker, D., Démoulin, P., Mandrini, C.H., DeRosa, M.L., Rouillard, A.P., Opitz, A., Stenborg, G., Vourlidas, A., Brooks, D.H.: 2012, Magnetic topology of active regions and coronal holes: Implications for coronal outflows and the solar wind. Solar Phys. 281, 237. DOI. ADS.

Verbeeck, C., Delouille, V., Mampaey, B., De Visscher, R.: 2014, The SPoCA-suite: Software for extraction, characterization, and tracking of active regions and coronal holes on EUV images. Astron. Astrophys. 561, A29. DOI. ADS.

Wang, Y.-M., Young, P.R., Muglach, K.: 2014, Evidence for two separate heliospheric current sheets of cylindrical shape during mid-2012. Astrophys. J. 780, 103. DOI. ADS.

Warren, H.P., Ugarte-Urra, I., Young, P.R., Stenborg, G.: 2011, The temperature dependence of solar active region outflows. Astrophys. J. 727, 58. DOI. ADS. 
Wilhelm, K., Curdt, W., Marsch, E., Schühle, U., Lemaire, P., Gabriel, A., Vial, J.-C., Grewing, M., Huber, M.C.E., Jordan, S.D., Poland, A.I., Thomas, R.J., Kühne, M., Timothy, J.G., Hassler, D.M., Siegmund, O.H.W.: 1995, SUMER - Solar Ultraviolet Measurements of Emitted Radiation. Solar Phys. 162, 189. DOI. ADS. 
Table 1. Summary table of the characteristics of the boxed regions within the ARs studied. The observed Doppler flows are defined as either 'Up', 'Down' or, if both blue and red shifts are seen, 'Mixed'. We also indicate whether the observed flows appear at the compact base of extended coronal loops (b) or in coronal loops (l). The behaviour of the photospheric magnetic field originating within each boxed region (CL: closed, OP: open, N-OP: narrow open) and the existence of any separatrix surfaces S.S. crossing the box or nearby (Nr.) is noted. The typical maximum height reached by the closed field is given in brackets. ${ }^{\dagger}$ Two $\mathrm{CHs}$ are observed nearby either side of the AR. ${ }^{\ddagger}$ Separatrix surface footprint is found at the base of these loops.

\begin{tabular}{|c|c|c|c|c|c|c|c|c|}
\hline \multirow{2}{*}{$\begin{array}{c}\text { Active } \\
\text { region } \\
\text { (EIS obs) }\end{array}$} & \multicolumn{6}{|c|}{ Doppler regions } & \multirow{2}{*}{$\begin{array}{c}\text { Underlying } \\
\text { B-field } \\
\text { height }(\mathrm{Mm})\end{array}$} & \multirow{2}{*}{$\begin{array}{l}\text { C.H. or } \\
\text { S.S. }\end{array}$} \\
\hline & No. & $\begin{array}{c}\text { Flow } \\
\text { (obs field) }\end{array}$ & $\begin{array}{l}\text { FIP } \\
\text { bias }\end{array}$ & $\begin{array}{c}T \\
(\mathrm{~K})\end{array}$ & $\begin{array}{c}n_{\mathrm{e}} \\
\left(\mathrm{cm}^{-3}\right)\end{array}$ & $\begin{array}{c}\text { EM } \\
\left(\mathrm{cm}^{-5}\right)\end{array}$ & & \\
\hline \multirow{3}{*}{$\begin{array}{c}\text { AR } 11271 \\
(21-A u g-11)\end{array}$} & 1 & $\mathrm{Up}(\mathrm{l})$ & 2.0 & 6.25 & 8.57 & 27.63 & CL(50) & Nr. S.S. ${ }^{\dagger}$ \\
\hline & 2 & $\mathrm{Up}(\mathrm{b}+\mathrm{l})$ & 1.6 & 6.20 & 8.46 & 27.37 & CL(150) & S.S. ${ }^{\dagger}$ \\
\hline & 3 & $\mathrm{Up}(\mathrm{b})$ & 1.8 & 6.20 & 8.47 & 27.48 & $\mathrm{OP}$ & Nr. S.S. ${ }^{\dagger}$ \\
\hline \multirow{3}{*}{$\begin{array}{c}\text { AR } 11419 \\
(17-F e b-12)\end{array}$} & 1 & $\mathrm{Up}(\mathrm{l})$ & 1.5 & 6.20 & 8.94 & 27.77 & $\mathrm{CL}(50)$ & S.S. \\
\hline & 2 & $\mathrm{Up}(\mathrm{b}+\mathrm{l})$ & 1.6 & 6.25 & 8.81 & 27.86 & $\mathrm{CL}(250)$ & S.S. \\
\hline & 3 & $\begin{array}{c}\text { Mixed } \\
(\mathrm{b}+\mathrm{l})\end{array}$ & 1.8 & 6.20 & 8.81 & 27.82 & $\mathrm{CL}(50)$ & S.S. \\
\hline \multirow{3}{*}{$\begin{array}{c}\text { AR } 11427 \\
(2-M a r-12)\end{array}$} & 1 & $\mathrm{Up}(\mathrm{l})$ & 1.6 & 6.25 & 8.75 & 27.90 & $\mathrm{CL}(50)$ & S.S. \\
\hline & 2 & Mixed(?) & 1.8 & 6.20 & 8.98 & 28.04 & $\mathrm{CL}(50)$ & S.S. \\
\hline & 3 & $\mathrm{Up}(\mathrm{b})$ & 2.1 & 6.25 & 8.44 & 27.27 & $\begin{array}{c}+\mathrm{N}-\mathrm{OP} \\
\mathrm{CL}(250) \\
+\mathrm{N}-\mathrm{OP}\end{array}$ & S.S. \\
\hline \multirow{2}{*}{$\begin{array}{c}\text { AR } 11445 \\
(28-M a r-12)\end{array}$} & 1 & $\mathrm{Up}(\mathrm{b})$ & 2.2 & 6.20 & 8.88 & 27.91 & $\mathrm{CL}(250)$ & S.S. \\
\hline & 2 & $\mathrm{Up}(\mathrm{b}+\mathrm{l})$ & 1.9 & 6.25 & 8.55 & 27.55 & $\begin{array}{l}\mathrm{CL}(250) \\
+\mathrm{N}-\mathrm{OP}\end{array}$ & S.S. \\
\hline \multirow{3}{*}{$\begin{array}{c}\text { AR } 11553 \\
(29-\text { Aug-12) }\end{array}$} & 1 & Down(?) & 3.7 & 6.25 & 8.96 & 27.94 & OP & S.S. \\
\hline & 2 & $\mathrm{Up}(\mathrm{l})$ & 1.9 & 6.90 & 8.55 & 27.73 & $\mathrm{OP}$ & S.S. \\
\hline & 3 & $\mathrm{Up}(\mathrm{l})$ & 1.6 & 6.25 & 8.53 & 27.54 & $\mathrm{CL}(250)$ & None ${ }^{\ddagger}$ \\
\hline \multirow[t]{2}{*}{$\begin{array}{c}\text { AR } 11569 \\
(15-\text { Sep-12) }\end{array}$} & 1 & $\mathrm{Up}(\mathrm{b})$ & 2.4 & 6.25 & 8.91 & 27.69 & $\mathrm{CL}(120)$ & $\begin{array}{l}\text { C.H. } \\
\text { S.S. }\end{array}$ \\
\hline & 2 & Down(b) & 2.2 & 6.20 & 9.18 & 27.72 & $\mathrm{OP}$ & $\begin{array}{l}\text { C.H. } \\
\text { S.S. }\end{array}$ \\
\hline \multirow{4}{*}{$\begin{array}{c}\text { AR } 11575 \\
(23-S e p-12)\end{array}$} & 1 & $\mathrm{Up}(\mathrm{l})$ & 1.7 & 6.25 & 8.72 & 27.49 & $\mathrm{CL}(50)$ & S.S. \\
\hline & 2 & $\mathrm{Up}(\mathrm{b}+\mathrm{l})$ & 2.0 & 6.40 & 8.68 & 27.44 & CL(50) & Nr. S.S. \\
\hline & 3 & Mixed(b) & 1.7 & 6.20 & 8.65 & 27.69 & CL(250) & None \\
\hline & 4 & $\begin{array}{l}\text { Mixed } \\
(\mathrm{b}+\mathrm{l})\end{array}$ & 1.9 & 6.25 & 8.90 & 28.26 & $\begin{array}{l}\mathrm{CL}(50) \\
+\mathrm{N}-\mathrm{OP}\end{array}$ & S.S. \\
\hline
\end{tabular}

